\title{
ESTUDIO DE PELIGRO SÍSMICO DE ECUADOR Y PROPUESTA DE ESPECTROS DE DISEÑO PARA LA CIUDAD DE CUENCA
}

\author{
Pablo Quinde Martínez ${ }^{(1)}$ y Eduardo Reinoso Angulo ${ }^{(2)}$
}

\begin{abstract}
RESUMEN
En este trabajo se presenta un estudio de peligro sísmico para el Ecuador. La falta de catálogos sísmicos completos, o en muchos casos la poca información relacionada al tema, hace que sea necesario un análisis probabilista de amenaza sísmica de manera que sea posible considerar las diferentes incertidumbres que se presentan en el proceso de cálculo. Esta metodología ha sido empleada en el presente trabajo para el estudio de peligro sísmico del Ecuador. Se debe partir de un catálogo que sea lo suficientemente depurado, homogéneo y completo, de manera que los parámetros sísmicos de las diferentes fuentes no presenten grandes desviaciones en sus resultados. Posteriormente, se estudiaron diferentes modelos de atenuación, los mismos que son necesarios para conocer las intensidades en cualquier punto de interés. En este estudio se obtienen resultados de peligro sísmico como curvas de peligro, espectros de peligro uniforme (EPU) y mapas de peligro sísmico, para diferentes periodos de retorno. Como aplicación de estos resultados, se obtienen espectros de diseño sísmico para la ciudad de Cuenca, partiendo de un estudio previo de efectos de sitio realizado por la Universidad de Cuenca en el año 2000.
\end{abstract}

Palabras clave: peligro sísmico; catálogo sísmico; espectros de peligro uniforme; curvas de peligro símico; espectros de diseño; efectos de sitio

\section{SEISMIC HAZARD ASSESSMENT FOR ECUADOR AND DESIGN SPECTRA PROPOSED FOR THE CITY OF CUENCA}

\begin{abstract}
A seismic hazard study for Ecuador is presented in this paper. The lack of complete seismic catalogs or the little information available, makes necessary a probabilistic seismic hazard analysis to include the various uncertainties that arise in the calculation process. This methodology has been used in this work for the study of seismic hazard of Ecuador. The catalog must be sufficiently refined, homogeneous and complete, so that the seismic parameters of the different sources do not exhibit large deviations in the results. Different attenuation models are presented, since they are
\end{abstract}

Artículo recibido el 29 de octubre de 2015 y aprobado para su publicación el 4 de abril de 2016. Se aceptarán comentarios y/o discusiones hasta cinco meses después de su publicación.

(1) Candidato a Doctorado, Instituto de Ingeniería de la Universidad Nacional Autónoma de México., Cd. Universitaria, Coyoacán, C:P: 04510, México, D.F., Tel. +52 (55) 56233500; PQuindeM@iingen.unam.mx,

(2) Investigador Titular, Instituto de Ingeniería de la Universidad Nacional Autónoma de México., Cd. Universitaria, Coyoacán, C:P: 04510, México, D.F., Tel. +52 (55) 56233500; EReinosoA@iingen.unam.mx 
necessary to estimate the intensities at any point of interest. In this study, seismic hazard results such as hazard curves, uniform hazard spectra (EPU) and seismic hazard maps for different return periods are presented. As a case study, seismic design spectra for Cuenca are obtained, based on a previous site effects study conducted by the University of Cuenca in the year 2000.

Keywords: seismic hazard; seismic catalog; uniform hazard spectra; seismic hazard curves; design spectra; site effects

\section{INTRODUCCIÓN}

El principal objetivo de un estudio de peligro sísmico es el de caracterizar, de la manera más precisa posible, los niveles de movimiento del terreno que deberá soportar una determinada estructura. Se busca que el nivel de demanda sísmica se encuentre asociado a un nivel de desempeño de la estructura determinado previamente, de manera que se puedan controlar los daños que ocasionaría el evento sísmico. Ahora, el reto importante recae en manejar las diferentes incertidumbres que se presentan en este fenómeno sísmico, como son, conocer la distancia, magnitud e intensidad del evento telúrico futuro adoptado como sismo de diseño. Es por esta falta de certeza en conocer sismos futuros que se utiliza un análisis probabilista de amenaza sísmica con la finalidad de tratar de predecir y modelar el comportamiento sísmico de la región de interés. La principal respuesta buscada sería determinar la probabilidad que la intensidad de cierto evento sísmico exceda una intensidad objetivo, la cual estaría relacionada con el nivel de desempeño estructural buscado.

Los criterios de diseño sismorresistente se basan en métodos probabilísticos que suponen las intensidades y frecuencias de ocurrencia de cierto evento sísmico. Debido a que no es posible contar con una base de datos tal que permita estimar con exactitud la ocurrencia de eventos sísmicos, se recurre a la teoría de probabilidades para considerar las incertidumbres en la estimación de su ocurrencia. Una manera de ilustrar los resultados del análisis probabilista de amenaza sísmica es a través de curvas de peligro, espectros de peligro uniforme o mapas de amenaza sísmica, que muestran valores tales como aceleraciones máximas probables para diferentes periodos de retorno. A partir de estos se pueden obtener resultados, tales que, sea posible plasmar un espectro de diseño en un código de construcción.

Ecuador se encuentra dentro del denominado Cinturón de Fuego del Pacífico, por lo que su actividad sísmica es alta, incluyendo eventos tales como el de 1906, que alcanzó una magnitud de 8.8, colocándolo dentro de los sismos más grandes de la historia. Salvo el sismo de Bahía de Caráquez en agosto de 1997 con una magnitud de 7.1, y que dejó en evidencia ciertos problemas relacionados al diseño sismorresistente en el Ecuador, en esta nación no han existido eventos catastróficos en más de un siglo, lo que ha provocado que no se le preste la atención debida a esta problemática. Si bien es cierto, existe una Norma Ecuatoriana de la Construcción, también lo es el hecho que los estudios acerca del tema en el país son escasos.

Se podrían citar los estudios de zonificación sísmica para el Ecuador realizados por Aguiar (2010), en el que se incluyen modelos de atenuación construidos a partir de información macrosísmica, aunque las fuentes sísmicas utilizadas no tienen el suficiente respaldo técnico. La Norma Ecuatoriana de la Construcción 2014 (NEC), en el capítulo 2, presenta una zonificación sísmica del Ecuador, y una breve descripción del estudio de peligro sísmico realizado. Por otra parte, García y Peñafiel (2000) realizaron un estudio de peligro sísmico, pero únicamente para la ciudad de Cuenca, en el que se utilizaron fuentes puntuales, agrupando los registros sísmicos. Un estudio más reciente realizado por Alvarado (2012), describe de manera detallada treinta fuentes sísmicas para Ecuador, además en los trabajos de Beauval et al. 2013 y 2014 se realizan estudios del catálogo sísmico del Ecuador y un estudio de peligro sísmico para la ciudad de Quito, respectivamente. 


\section{TECTÓNICA Y SISMICIDAD DEL ECUADOR}

\section{Breve descripción de la tectónica del Ecuador}

El territorio ecuatoriano se encuentra conformado por una región continental, la cual va desde $81^{\circ} \mathrm{W}$ hasta $75^{\circ} \mathrm{W}$ en longitud, y $1.25^{\circ} \mathrm{N}$ hasta $5^{\circ} \mathrm{S}$ en latitud, aproximadamente, y una parte insular conformada por las islas Galápagos. El territorio se divide en cuatro regiones, Costa, Sierra, Oriente y Región Insular, siendo la segunda en donde existen la mayor cantidad de fallas debido a los pliegues que se producen por la geodinámica de la región.

Ecuador se encuentra dentro de un particular movimiento tectónico. Un sector del territorio forma parte de la microplaca denominada "Bloque andino", la cual pertenece a la placa sudamericana. Esta microplaca, se encuentra en una interacción entre las placas de Nazca, Cocos y Caribe. El movimiento en esta zona se ha medido en un intervalo aproximado de 50-60 mm/año, mientras que para la placa continental, el movimiento se encuentra entre 2 y $5 \mathrm{~mm} / a n ̃ o$. Por otra parte, la región sur del Ecuador, se encuentra en interacción entre la placa Sudamericana y la placa de Nazca. Esta dinámica de placas ha producido que en el Ecuador se presenten tres diferentes inclinaciones de la subducción de la placa de Nazca en la placa Continental (Taipe, 2013).

a) De la latitud $1^{\circ} \mathrm{N}$ hacia el norte del territorio, se presenta una subducción normal con un ángulo aproximado de inclinación de $35^{\circ}$

b) Entre la brecha conformada por las latitudes $1^{\circ} \mathrm{N}$ y $3^{\circ} \mathrm{S}$, existe una inclinación de la subducción de $25^{\circ}$ aproximadamente

c) La tercera zona de inclinación de la subducción que se forma, al sur de la latitud $3^{\circ} \mathrm{S}$, presenta un ángulo menor que $25^{\circ}$

Este comportamiento dinámico de la región ha generado un sistema de fallas denominado Sistema Mayor Dextral (Alvarado, 2012), el mismo que presenta una mayor concentración de fallas y pliegues en la región de la sierra ecuatoriana. Este sistema de fallas, en conjunto con la subducción presentada en la costa del país, representan las principales fuentes generadores de sismos.

\section{Zonas sísmicas del Ecuador}

El peligro sísmico en Ecuador está regido principalmente por dos tipos de fuentes sísmicas: subducción (interplaca e intraplaca), y de tipo corticales (superficiales). En cada una de estas fuentes se lleva a cabo un proceso de acumulación y liberación de energía independiente del que ocurre en las demás fuentes. Los sismos interplaca (profundidad $<40 \mathrm{~km}$ ) corresponden a los sismos que se generan por el roce entre la placa de Nazca que subduce bajo la placa Sudamericana a lo largo de su zona de convergencia. Los sismos intraplaca (profundidad entre 40 y $300 \mathrm{~km}$ ) corresponden a los sismos de falla normal de profundidad intermedia, localizados dentro de la placa oceánica de Nazca que subduce bajo la placa continental Sudamericana. Los sismos corticales (profundidad $<40 \mathrm{~km}$ ) corresponden a los sismos someros que ocurren dentro de la placa Sudamericana.

En este trabajo, para modelar la geometría de las fuentes sísmicas de Ecuador, se utilizó como referencia el trabajo realizado por Alvarado (2012). Las fuentes sísmicas adoptadas en esta investigación, corresponden a diez del tipo subducción, y veinte superficiales o corticales. La geometría de estas fuentes (Alvarado, 2012) se observa en las figuras 1 y 2, mientras que ciertas características de las mismas se exponen en las tablas 1 y 2 . 


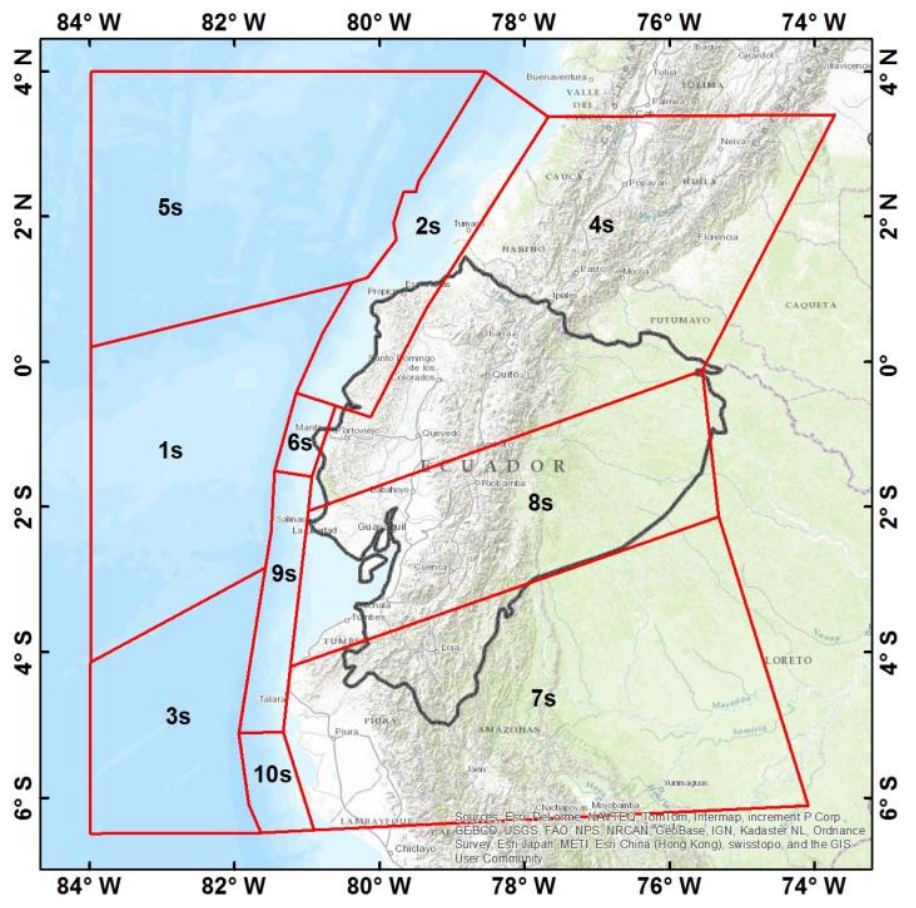

Figura 1. Fuentes sísmicas de tipo subducción (Alvarado, 2012)

Tabla 1. Fuentes de subducción

\begin{tabular}{ccc}
\hline ID & Nombre & Tipo de falla \\
\hline $1 \mathrm{~s}$ & Carnegie & Indeterminado \\
$2 \mathrm{~s}$ & Tumaco-Esmeraldas & Interplaca \\
$3 \mathrm{~s}$ & Grijalva & Indeterminado \\
$4 \mathrm{~s}$ & Ibarra & Intraplaca \\
$5 \mathrm{~s}$ & Malpelo & Indeterminado \\
$6 \mathrm{~s}$ & Manta & Interplaca \\
$7 \mathrm{~s}$ & Moyobamba & Intraplaca \\
$8 \mathrm{~s}$ & Puyo & Intraplaca \\
$9 \mathrm{~s}$ & Salinas & Interplaca \\
$10 \mathrm{~s}$ & Sechura & Interplaca \\
\hline
\end{tabular}

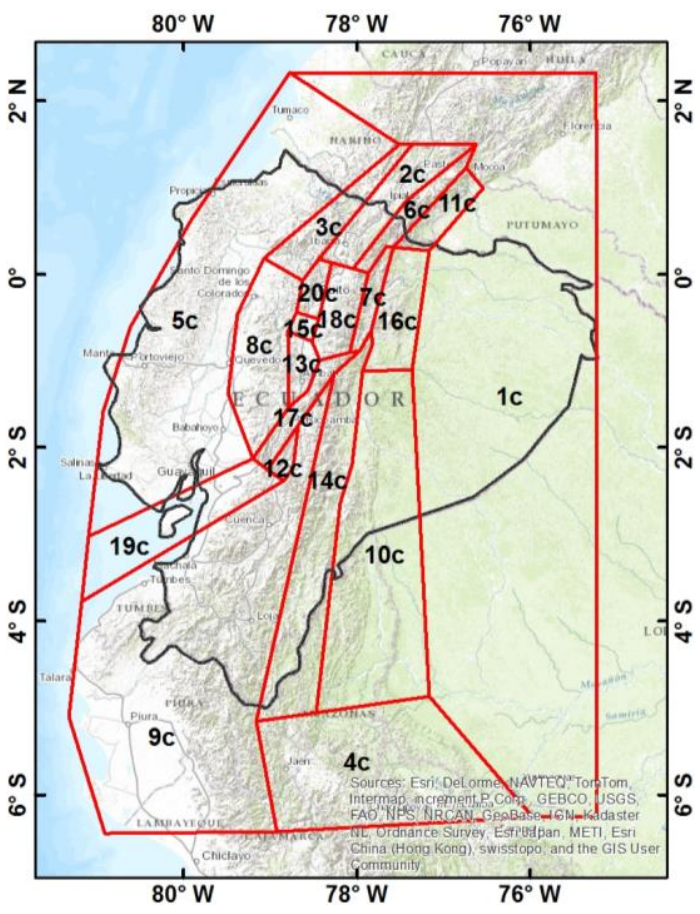

Figura 2. Fuentes sísmicas tipo corticales (Alvarado, 2012)

Tabla 2. Fuentes corticales

\begin{tabular}{ccc}
\hline ID & Nombre & Tipo de falla \\
\hline $1 \mathrm{c}$ & Amazonas & Indeterminado \\
2c & El Ángel-San Gabriel & Inversa y normal \\
$3 \mathrm{c}$ & Apuela-Huayrapungo & Normal \\
$4 \mathrm{c}$ & Bagua & Inversa \\
$5 \mathrm{c}$ & Canadé-San Lorenzo & Indeterminado \\
$6 \mathrm{c}$ & Chingual & Normal \\
$7 \mathrm{c}$ & Cofanés-Cosanga & Inversa \\
$8 \mathrm{c}$ & Cordillera-Occidental & Indeterminado \\
$9 \mathrm{c}$ & Cuenca Amotape & Indeterminado \\
$10 \mathrm{c}$ & Cutucú & Inversa \\
$11 \mathrm{c}$ & Sistema Frontal Este & Normal \\
$12 \mathrm{c}$ & Guamote & Inversa y normal \\
$13 \mathrm{c}$ & Latacunga & Inversa \\
$14 \mathrm{c}$ & Macas & Inversa \\
$15 \mathrm{c}$ & Machachi & Normal \\
$16 \mathrm{c}$ & Napo & Inversa \\
$17 \mathrm{c}$ & Pallatanga-Pisayambo & Normal \\
$18 \mathrm{c}$ & Papallacta & Indeterminado \\
$19 \mathrm{c}$ & Puná & Normal \\
$20 \mathrm{c}$ & Quito & Inversa \\
\hline
\end{tabular}




\section{CATÁlOGO SÍSMICO}

El estudio y creación de un correcto catálogo sísmico es un paso primordial para el estudio de peligrosidad sísmica de una región. De éste depende el correcto análisis estadístico de sismicidad de cada una de las fuentes. Se debe emplear una base de datos tan homogénea y completa como sea posible.

El catálogo compilado en este artículo corresponde aproximadamente a cinco siglos de recolección de datos, en el que se incluyen sismos de carácter histórico. La recopilación de este catálogo se llevó a cabo por Beauval et al. (2013).

Los catálogos estudiados para llegar a una compilación final fueron los del Instituto Geofísico de la Escuela Politécnica Nacional (IGEPN), de la Red Nacional de Sismógrafos del Ecuador (RENSIG), del National Earthquake Information Center (NEIC-PDE de la U.S. Geological Survey), de la International Seismological Center (ISC), de la Global Centroid Moment Tensor (GCMT), el catálogo Centennial compilado por Engdhal y Villaseñor (2002) y del EHB-ISC (versión más detallada del ISC), dando una mayor prioridad a los catálogos del IGEPN y de la RENSIG, ya que son regionales. Estas bases de datos incluyen eventos históricos e instrumentales, sin embargo, para los estudios posteriores se han separado estas dos etapas de datos, ya que los resultados pueden verse afectados debido a su diferencia de homogeneidad. El número total de eventos existentes en el catálogo instrumental es de 10823 eventos, que abarcan un periodo desde 1901 hasta 2009.

\section{Homogenización del catálogo}

Los tipos de magnitudes que se encontraron en los catálogos corresponden a magnitudes de ondas internas $\left(M_{b}\right)$, ondas superficiales $\left(M_{s}\right)$ y ondas de Coda $\left(M_{D}\right)$ que corresponde a la RENSIG (Palacios y Yepes, 2011). Así mismo, se encontraron magnitudes de tipo desconocidas $\left(M_{U K}\right)$ y asumidas a un evento $(M)$.

Las expresiones utilizadas para homogenizar estos diferentes tipos de magnitudes a una magnitud de tipo $M w$, son las siguientes:

- $\quad$ Magnitudes de ondas internas $\left(M_{b}\right)$ (Beauval, et. al, 2013)

$M w=0.93 M_{b}+0.6 \quad$ para $M_{b}<6$

Esta misma expresión fue utilizada para magnitudes de ondas tipo $M_{D}$.

A las magnitudes $M_{b}$ y $M_{D}$ mayores a 6, se les tomó similares a $M_{w}$.

- $\quad$ Magnitudes de ondas superficiales $\left(M_{S}\right)$ (Cahuari y Tavera, 2007),

$M w=0.7044 M_{s}+1.702$ para $4.5 \leq M_{s}<6.8$

A las magnitudes $M_{s}$ mayores a 6.8 , se les tomó similares a $M_{w}$.

- Los tipos de magnitudes de ondas del tipo $M_{U k}$ y $M$, han sido asumidos como $M_{w}$. Se encontraron 22 eventos con magnitud de tipo $M_{U k}$, de los cuales solo un evento tiene magnitud inferior a $6.0 \mathrm{y}$ 15 presentan magnitudes superiores a 6.8. El asumir directamente $M_{U k}=M_{w}$ no implicaría algún impacto significativo dentro del análisis de peligro sísmico, ya que de acuerdo a las expresiones 
de homogenización planteadas, para magnitudes superiores a 6.0, los cambios no son significativos. Lo mismo sucede con la magnitud del tipo $\mathrm{M}$, ya que solo existe un evento asociado a dicha magnitud y corresponde al evento de 1906 que registró una magnitud de 8.8.

\section{Discriminación de réplicas y premonitores}

En cuanto a la depuración del catálogo sísmico, el principal punto a considerar es la discriminación de réplicas y premonitores ya que solo se consideran los eventos principales para el cálculo de la sismicidad de cada fuente. Las réplicas afectan la distribución de los sismos con respecto al tiempo.

Si bien no existe un concepto general y específico para definir una réplica, en lo que si se concuerda, es que se encuentran definidas en eventos asignados a un espacio y un tiempo posteriores a un evento principal. Para encontrar las réplicas se utilizaron las expresiones de Maeda (1996), en las cuales se considera el decaimiento exponencial en número y magnitud de réplicas.

Longitud: $\mathrm{L} \leq 10^{(0.5 * M m-1.8)}$

Tiempo: $t \leq 10^{\frac{(0.17+0.85 *(M m-4))}{1.3}}-0.3$

donde, L corresponde a la distancia epicentral respecto al evento principal, $t$ el tiempo en días respecto al evento principal y $\mathrm{Mm}$ representa la magnitud del evento. Al analizar el catálogo utilizando esta metodología, se obtuvieron 14 por ciento de réplicas.

\section{Completez del catálogo sísmico}

Las tasas de recurrencia pueden variar debido a datos inconsistentes o incompletos del catálogo, lo que afecta a los parámetros sísmicos que se obtienen para cada una de las fuentes. Para estudiar los periodos en los que se puede considerar al catálogo completo, en este trabajo se estudiaron el método gráfico y el método de Stepp (1972).

El método gráfico permite observar la magnitud mínima de completez, la misma que define el límite inferior, a partir del cual, el catálogo se considera completo. En este método se analiza la frecuencia de ocurrencia acumulada y normalizada para cada intervalo de magnitud. En la figura 3 se observan los resultados obtenidos de todo el catálogo para dos intervalos de magnitudes.

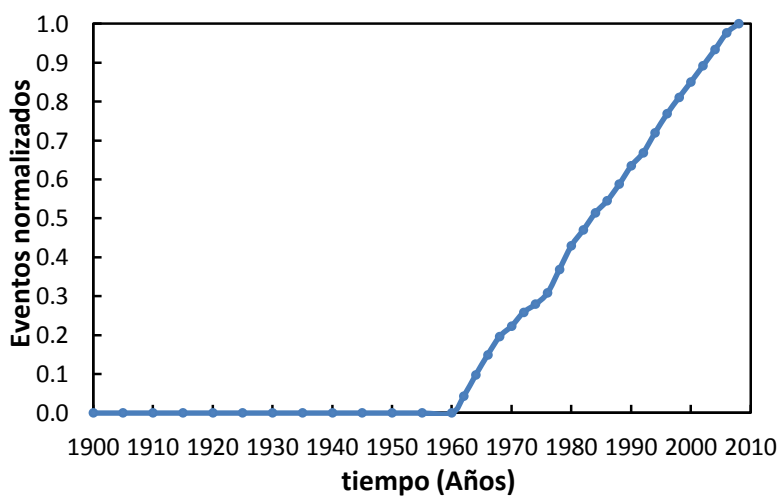

a)

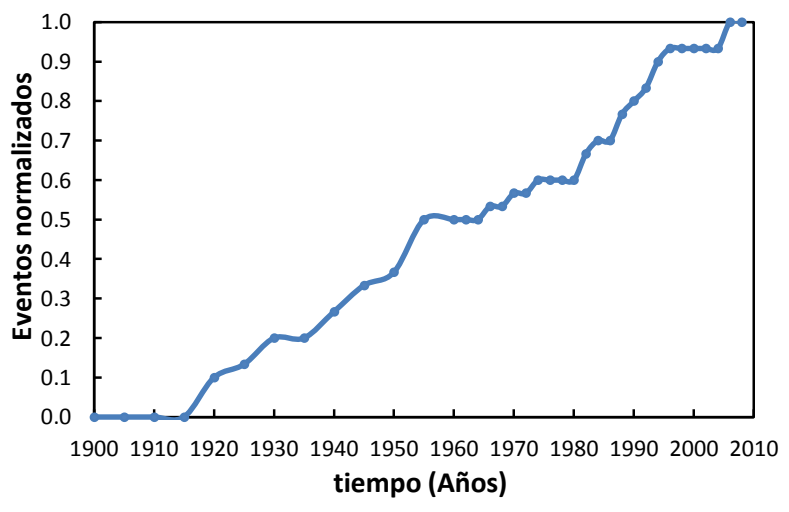

b)

Figura 3. Completez del catálogo por el método gráfico. a) Intervalo de magnitud entre 4.5 a 5.0, y b) Intervalo de magnitud entre 6.5 a 7.0 
El año a partir del cual la pendiente es constante se considera que el catálogo es completo para esa magnitud.

Por otra parte, el método de Stepp presenta un concepto similar en cuanto al análisis de la pendiente, pero en este caso se analiza el comportamiento de la desviación estándar (SR), a través del tiempo, para diferentes intervalos de magnitudes. La comparación de la pendiente de los datos de magnitudes se compara con la recta $1 / \sqrt{T_{i}}$, donde $T_{i}$ corresponde al tiempo transcurrido en años. En la figura 4 se observan los resultados obtenidos de todo el catálogo para los mismos intervalos de magnitudes presentados en el método gráfico.

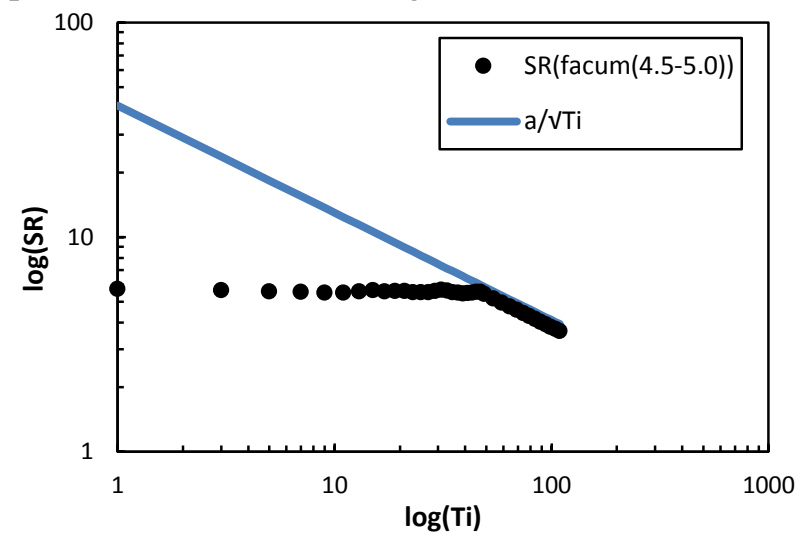

a)

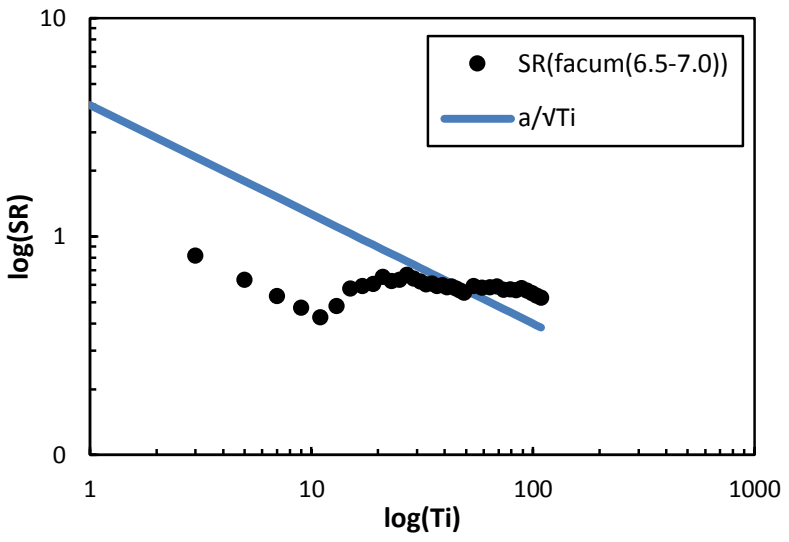

b)

Figura 4. Completez del catálogo sísmico por el método de Stepp. a) Intervalo de magnitud entre 4.5 a 5.0, y b) Intervalo de magnitud entre 6.5 a 7.0. Los puntos representan la desviación estándar (SR) de la frecuencia acumulada para el intervalo de magnitud especificado

En el método de Stepp se considera que el catalogo es completo cuando la desviación estándar sigue la pendiente de la recta $1 / \sqrt{T_{i}}$. La diferencia en la completez de los dos intervalos de magnitudes mostrados en las figuras 3 y 4 se debe principalmente al tamaño de la magnitud, ya que a partir de la instalación de la red mundial de sismógrafos estándar (WWSSN) en 1964, fue posible empezar a detectar sismos con magnitudes pequeñas $(\mathrm{Mw}<5)$, por lo que antes de este año los registros más comunes son de magnitudes grandes $(\mathrm{Mw}>6)$.

Este análisis de completez es primordial para la correcta caracterización de las fuentes, debiendo hacerse este estudio para cada una de las 30 fuentes estudiadas.

\section{DEMANDA SÍSMICA}

Se podría definir al peligro o amenaza sísmica como la probabilidad de que en un sitio de interés se excedan ciertos valores de intensidad en un lapso dado; es decir, conocer la frecuencia y severidad de los posibles eventos.

Debido a que las incertidumbres para predecir la ocurrencia de un evento sísmico son muy elevadas, y no se cuenta con un catálogo lo suficientemente extenso y completo como para predecir con precisión la ocurrencia de un evento sísmico con cierta magnitud, se realizó un análisis probabilístico de amenaza sísmica (PSHA, por sus siglas en inglés). 
La ventaja de este modelo de análisis probabilista, es que el estudio no se centra en un solo evento "crítico" y, por el contrario, involucra las incertidumbres (distancia, magnitud, entre otros) en la predicción de los niveles de intensidades sísmicas. Este análisis considera todos los eventos posibles, incluyendo la probabilidad de ocurrencia de cada uno de ellos. Si bien el PSHA incrementa la complejidad en los cálculos, también anexa herramientas de las cuales se pueden controlar y justificar, de una mejor manera, los resultados de peligro sísmico.

\section{Caracterización de las fuentes sísmicas}

Se analizaron la geometría, distancia fuente-sitio y sismicidad de las fuentes símicas. La geometría de las fuentes es de tipo área y corresponden a lo expuesto en las figuras 1 y 2.

\section{Distancia fuente-sitio}

La distancia que existe desde la fuente al sitio de interés se encuentra asociada al tipo de fuente definido previamente, pudiendo ser puntual, lineal o de tipo área. En la figura 5 se pueden observar las características de los diferentes tipos de distancias (Leonardo, 2012).
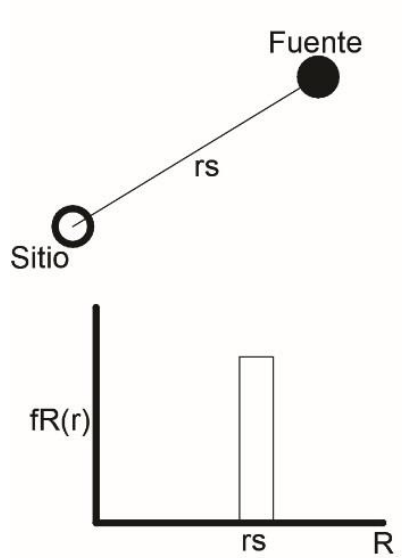

(a)
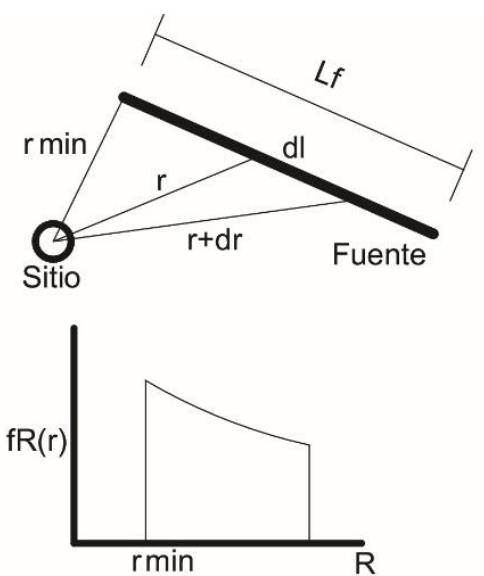

(b)
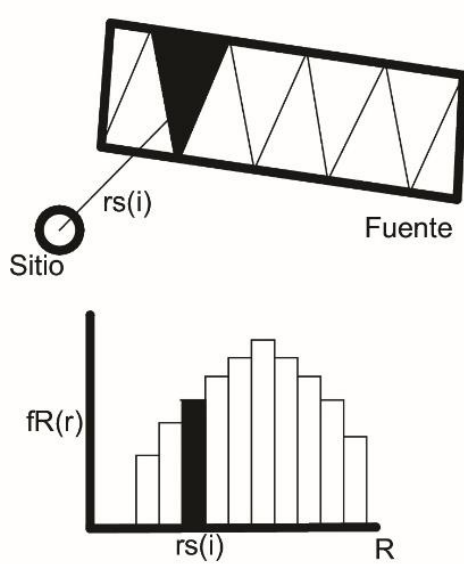

(c)

Figura 5. Tipos de fuentes y distancias fuente-sitio. (a) Fuente y distancia puntual, (b) fuente y distancia lineal y (c) fuente y distancia tipo área

Como se definió anteriormente, todas las fuentes, incluyendo su distancia al sitio, han sido consideradas como de tipo área, similares a la figura 5(c).

\section{Sismicidad de las fuentes}

Un paso fundamental en un estudio tipo PSHA es conocer la frecuencia y severidad con la que se podrían presentar los eventos sísmicos dentro de una misma fuente. Para esto, se puede considerar que los sismos tienen las misma probabilidad de ocurrencia dentro de la geometría de la fuente, y que su recurrencia (magnitud vs frecuencia) observada en los catálogos sísmicos es suficiente para predecir los eventos en cualquier lapso.

El primer paso es el de conocer la tasa de excedencia de intensidades, representada normalmente por $\lambda(M)$. Analizando el catálogo sísmico se puede tratar de intuir la distribución de magnitudes de los eventos principales, la cual, conceptualmente sigue la relación recurrencia de Gutenberg-Richter (GR) (Gutenberg y Richter, 1944), mostrada en la ecs. 5 y 6 : 
$\log \lambda(M)=a+b M$

$\lambda(M)=e^{\alpha-\beta M}$

donde $\lambda(M)$ es la tasa media anual de excedencia de un sismo con magnitud $M$, por otra parte $\alpha=a \ln (10)$ y $\beta=b \ln (10)$. Los parámetros $a$ y $b$ son generalmente obtenidos mediante el método de máxima verosimilitud, y representan la tasa global de sismos en la región y la probabilidad relativa de ocurrencia entre eventos con magnitudes altas y bajas, respectivamente. La ec. 6 cubre un intervalo de ($\infty,+\infty)$; sin embargo, el interés recae en un intervalo que represente los eventos con magnitudes que pudieran afectar a las estructuras, este intervalo se podría representar como $\left[M_{0}, M_{u}\right]$, cuyos valores quedarían definidos por los catálogos sísmicos.

Si se eliminan los eventos con magnitud $M<M_{0}$, la tasa de excedencia puede representarse de la siguiente manera:

$\lambda(M)=v \cdot e^{-\beta\left(M-M_{0}\right)} \rightarrow \operatorname{para} M>M_{0}$

donde $v=e^{\alpha-\beta M_{0}}$ es la tasa media anual de excedencia y $M_{0}$ es la magnitud umbral elegida. La distribución de probabilidad de magnitud del modelo de recurrencia de Gutenberg-Richter, con límite inferior, se puede expresar en términos de la función de distribución acumulativa (CDF, por sus siglas en inglés) o en su respectiva función de densidad de probabilidad (PDF, por sus siglas en inglés), tal como en las ecs. 8 y 9 , respectivamente:

$F_{M}(M)=P\left[m<M \mid m>M_{0}\right]=\frac{\lambda_{M_{0}}-\lambda_{M}}{\lambda_{M_{0}}}=v\left[1-e^{-\beta\left(M-M_{0}\right)}\right]$

$f_{M}(M)=\frac{d}{d M} F_{M}(M)=v \beta e^{-\beta\left(M-M_{0}\right)}$

Cada fuente sísmica está asociada a una magnitud máxima (McGuire, 1995), por lo que en el otro extremo de la escala de magnitudes $(M \rightarrow+\infty)$ se debería truncar la PDF en una magnitud máxima $M_{u}$. El modelo truncado de Gutenberg-Richter (Cornell y Vanmarcke, 1969) tiene una tasa anual de excedencia que puede ser calculada mediante la siguiente expresión:

$\lambda(M)=v\left[\frac{e^{-\beta\left(M-M_{0}\right)}-e^{-\beta\left(M_{u}-M_{0}\right)}}{1-e^{-\beta\left(M_{u}-M_{0}\right)}}\right] ;$ para $M_{0} \leq M \leq M_{u}$

donde $\lambda(M)$ es la tasa anual de excedencia, $M_{0}$ y $M_{u}$ representan las magnitudes mínimas y máximas consideradas dentro del catálogo completo de cada fuente, $v$ y $\beta$ son parámetros que definen la tasa de excedencia de cada una de las fuentes sísmicas, los cuales se estiman mediante procesos estadísticos de la información sobre la sismicidad de la fuente.

Aparte de estudiar las incertidumbres debido a la magnitud y ubicación de los eventos sísmicos, también se deben considerar las incertidumbres temporales, generalmente representadas como un comportamiento "Poissoniano".

El modelo de Poisson asume el tiempo entre dos sismos como una variable con distribución exponencial, siendo estos eventos independientes en el tiempo. La probabilidad de ocurrencia de un sismo 
en una ventana de tiempo está relacionada únicamente al tamaño de la ventana, y es totalmente independiente inclusive del tiempo transcurrido desde el sismo más reciente (Baker, 2015). Podría existir una probabilidad de ocurrencia de más de un sismo dentro de la ventana de tiempo, pero esta probabilidad es despreciable. La función de probabilidad de Poisson se puede expresar de la siguiente manera:

$P(N=n)=\frac{e^{-\lambda(M) t}\left[e^{-\lambda(M) t}\right]^{n}}{n !}$

Además de los modelos mencionados, se utilizó una estimación por máxima verosimilitud para encontrar los parámetros sísmicos de cada una de las fuentes sísmicas (McGuire, 2004). Dichos parámetros se muestran en la tabla 3.

Tabla 3. Valores de los parámetros símicos para cada fuente

\begin{tabular}{cccccc|ccccccc}
\hline Fuente & $\mathbf{M}_{\mathbf{0}}$ & $\boldsymbol{\lambda}_{\mathbf{0}}$ & $\mathbf{E}(\boldsymbol{\beta})$ & $\mathbf{c}(\boldsymbol{\beta})$ & $\mathbf{E}(\mathbf{M u})$ & Fuente & $\mathbf{M}_{\mathbf{0}}$ & $\boldsymbol{\lambda}_{\mathbf{0}}$ & $\mathbf{E}(\boldsymbol{\beta})$ & $\mathbf{c}(\boldsymbol{\beta})$ & $\mathbf{E}(\mathbf{M u})$ \\
\hline Fuente 1s & 4.00 & 0.91 & 3.93 & 0.20 & 6.40 & Fuente $6 \mathrm{c}$ & 4.00 & 0.11 & 1.02 & 0.70 & 6.20 \\
Fuente 2s & 4.00 & 2.01 & 1.34 & 0.07 & 8.80 & Fuente $7 \mathrm{c}$ & 4.50 & 0.14 & 1.12 & 0.30 & 6.80 \\
Fuente 3s & 4.20 & 0.48 & 1.41 & 0.23 & 6.50 & Fuente $8 \mathrm{c}$ & 4.30 & 0.45 & 2.53 & 0.25 & 6.40 \\
Fuente 4s & 4.10 & 2.95 & 1.75 & 0.09 & 7.30 & Fuente $9 \mathrm{c}$ & 4.50 & 2.57 & 2.01 & 0.07 & 7.50 \\
Fuente 5s & 4.20 & 2.97 & 2.69 & 0.06 & 6.50 & Fuente $10 \mathrm{c}$ & 4.20 & 3.11 & 2.49 & 0.07 & 7.10 \\
Fuente 6s & 4.80 & 0.62 & 1.79 & 0.30 & 7.60 & Fuente $11 \mathrm{c}$ & 4.30 & 0.10 & 4.61 & 0.57 & 5.80 \\
Fuente 7s & 4.50 & 6.95 & 2.45 & 0.04 & 7.50 & Fuente $12 \mathrm{c}$ & 4.00 & 0.10 & 1.65 & 0.50 & 6.30 \\
Fuente 8s & 4.50 & 5.70 & 2.32 & 0.04 & 7.30 & Fuente $13 \mathrm{c}$ & 4.50 & 0.20 & 2.14 & 0.30 & 6.70 \\
Fuente 9s & 4.20 & 0.88 & 1.51 & 0.14 & 8.00 & Fuente $14 \mathrm{c}$ & 4.50 & 0.87 & 2.40 & 0.19 & 6.80 \\
Fuente 10s & 4.30 & 0.94 & 1.32 & 0.14 & 8.10 & Fuente $15 \mathrm{c}$ & 4.00 & 0.10 & 1.80 & 0.57 & 6.10 \\
Fuente 1c & 4.50 & 0.70 & 2.57 & 0.11 & 7.40 & Fuente $16 \mathrm{c}$ & 4.50 & 0.42 & 1.96 & 0.30 & 6.60 \\
Fuente 2c & 4.00 & 0.53 & 3.58 & 0.33 & 6.80 & Fuente $17 \mathrm{c}$ & 4.50 & 0.24 & 2.05 & 0.26 & 7.30 \\
Fuente 3c & 4.50 & 0.23 & 1.95 & 0.35 & 6.10 & Fuente $18 \mathrm{c}$ & 4.20 & 0.25 & 2.30 & 0.44 & 6.40 \\
Fuente 4c & 4.50 & 2.16 & 1.97 & 0.09 & 7.40 & Fuente $19 \mathrm{c}$ & 4.50 & 0.72 & 2.10 & 0.12 & 6.80 \\
Fuente 5c & 4.50 & 1.26 & 2.31 & 0.13 & 7.20 & Fuente $20 \mathrm{c}$ & 4.10 & 0.44 & 2.65 & 0.30 & 6.40 \\
\hline
\end{tabular}

s: corresponde a fuentes de subducción

c: corresponde a fuentes corticales

\section{Ecuaciones predictivas del movimiento del suelo}

Para conocer el decaimiento de las ondas sísmicas, desde la fuente al sitio específico se requiere el uso de modelos de atenuación, los mismos que relacionan magnitud, posición relativa fuente-sitio, profundidad, entre otros. Una manera general de representar estos modelos de atenuación es la siguiente.

$A=f(M, R, d$, tipo de suelo, mecanismo focal $\ldots)$

donde, $M$ representa la magnitud del evento, $R$ la distancia entre el evento y la fuente y $d$ la profundidad a la que ocurre el sismo.

Estas funciones se obtienen a partir de un análisis de regresión de los niveles de intensidad máxima del suelo (aceleración, velocidad, desplazamiento), contra la magnitud y distancia hipocentral de los eventos registrados. Los parámetros anteriores son obtenidos de registros acelerográficos de diversos sitios con características similares. Dado que la base de datos utilizada para obtener estos modelos no es muy grande ni completa, lo que se obtiene representa el promedio. Debido a estas incertidumbres y falta de información se puede modelar a la intensidad sísmica como una variable aleatoria de distribución lognormal, con mediana dada por el modelo de atenuación y desviación estándar del logaritmo natural igual a $\sigma_{\ln (a)}($ Ordaz et al., 1997). 
$\operatorname{Pr}(A>a \mid M, R)=\Phi\left[\frac{E(\ln (a) \mid M, R)-\ln (a)}{\sigma_{\ln (a)}}\right]$

donde, $\Phi($.$) , es la distribución normal estándar acumulada, E(\ln (a) \mid M, R)$, corresponde el valor esperado del logaritmo de la intensidad en relación a la ley de atenuación correspondiente, y $\sigma_{\ln (a)}$ es la correspondiente desviación estándar del logaritmo de $a$. Los modelos de atenuaciones utilizados en este trabajo fueron, Abrahamson y Silva (2008) para sismos corticales y Youngs et al. (1997) para sismos de subducción interplaca e intraplaca (ver tabla 4).

Tabla 4. Características de los modelos de atenuación utilizados

\begin{tabular}{cccccc}
\hline Nombre & $\begin{array}{c}\text { Intervalo de } \\
\text { periodo espectral } \\
(\mathbf{s})\end{array}$ & $\begin{array}{c}\text { Intervalo de } \\
\text { distancia válido } \\
(\mathbf{k m})\end{array}$ & $\begin{array}{c}\text { Intervalo de } \\
\text { magnitud válido } \\
\left(\mathbf{M}_{\mathbf{w}}\right)\end{array}$ & $\begin{array}{c}\text { Tipo de medida } \\
\text { de distancia }\end{array}$ & $\begin{array}{c}\text { Región } \\
\text { tectónica }\end{array}$ \\
\hline $\begin{array}{c}\text { Abrahamson \& } \\
\text { Silva (2008) } \\
\text { Youngs et al., } \\
\text { (1997) }\end{array}$ & $0.01-5.0$ & $0.01-200$ & $4.0-7.5$ & $\begin{array}{c}\text { Distancia a la } \\
\text { ruptura } \\
\text { Distancia a la } \\
\text { ruptura }\end{array}$ & $\begin{array}{c}\text { Corteza } \\
\text { Superficial }\end{array}$ \\
\hline
\end{tabular}

El modelo de atenuación de Abrahamson y Silva (2008) fue resultado del análisis de 2675 registros, correspondientes a 129 sismos superficiales originados en distintas regiones sísmicas activas a nivel mundial. El modelo de atenuación de Youngs et al. (1997) fue generado después de que los autores analizaron datos correspondientes a sismos de subducción interplaca e intraplaca con $\mathrm{M}_{\mathrm{w}} \geq 5$, registrados en estaciones localizadas en roca situadas en campo libre, a un distancia entre 10 y $500 \mathrm{~km}$. La utilización de estos modelos de atenuación se basó en las recomendaciones hechas por el Global Earthquake Model (GEM) y un estudio de validación de diversas Ecuaciones de Predicción de Movimiento de Suelo (GMPE's, por sus siglas en inglés) que fueron generadas en ambientes tectónicos similares a los de Ecuador, realizado por Taipe (2013), en donde se muestra que los modelos utilizados representan de manera razonable el movimiento de ondas sísmicas en la región. No se pudo realizar un estudio más específico ya que no se tuvo acceso a registros acelerográficos del Ecuador.

\section{Cálculo del peligro sísmico}

Para realizar el cálculo del peligro sísmico de una determinada región es necesario haber definido las fuentes y sus respectivos parámetros de sismicidad, además de los modelos de atenuación de onda para cada una de ellas. La amenaza sísmica puede calcularse considerando la suma de los efectos de cada una de las fuentes sísmicas y la distancia entre cada fuente y el sitio de interés. El peligro sísmico, expresado en función de la tasa de excedencia de intensidades, $v(a)$, se calcula mediante la ec. 14 (Esteva, 1970):

$$
v(a)=\sum_{i=1}^{N f} \lambda_{0 i} \int_{M_{0}}^{M_{u}} \int_{R_{0 i}}^{R_{u i}} \operatorname{Pr}\left(A>a \mid M, R_{i}\right) f_{m i}(M) f_{R i}\left(R_{i}\right) d M d R
$$

donde $\lambda_{0 i}$ es la tasa anual de excedencia de los eventos sísmicos con $M \geq M_{0}$ en la i-ésima fuente; $f_{m i}(M)$ y $f_{R i}\left(R_{i}\right)$ son funciones de densidad de probabilidad de la magnitud $(M)$ y distancia $(R)$ de la iésima fuente; $R_{0 i}$ y $R_{u i}$ representan el rango de distancias del evento a la i-ésima fuente; $\operatorname{Pr}(A>$ $\left.a \mid M, R_{i}\right)$, es la probabilidad de que la intensidad exceda un cierto valor, dada la magnitud del sismo, $M, \mathrm{y}$ la distancia entre la $i$-ésima fuente y el sitio $R_{i}$. Para el cálculo del peligro sísmico, se utilizó el programa CRISIS2015 Ver 1.0, desarrollado en el Instituto de Ingeniería de la UNAM (Ordaz et al., 2015), el cual utiliza una metodología probabilística para el cálculo de los resultados de peligro sísmico. 


\section{RESULTADOS DEL ESTUDIO DE PELIGRO SÍSMICO}

Los resultados del estudio de peligro sísmico del Ecuador están presentados a través de curvas de peligro sísmico, espectros de peligro uniforme y mapas de peligro sísmico. Los resultados, con excepción de los mapas, corresponden a las capitales de las seis provincias de mayor relevancia del Ecuador. No se presentan los resultados para todo el territorio ya que la información es muy extensa.

\section{Curvas de peligro sísmico}

En la figura 6 se muestran las curvas de peligro sísmico para la aceleración máxima del suelo (PGA, por sus siglas en inglés) y pseudoaceleración para periodos estructurales de $0.1 \mathrm{~s}, 0.2 \mathrm{~s}, 0.5 \mathrm{~s}$ y $1 \mathrm{~s}(\mathrm{Sa}(\mathrm{g}))$.
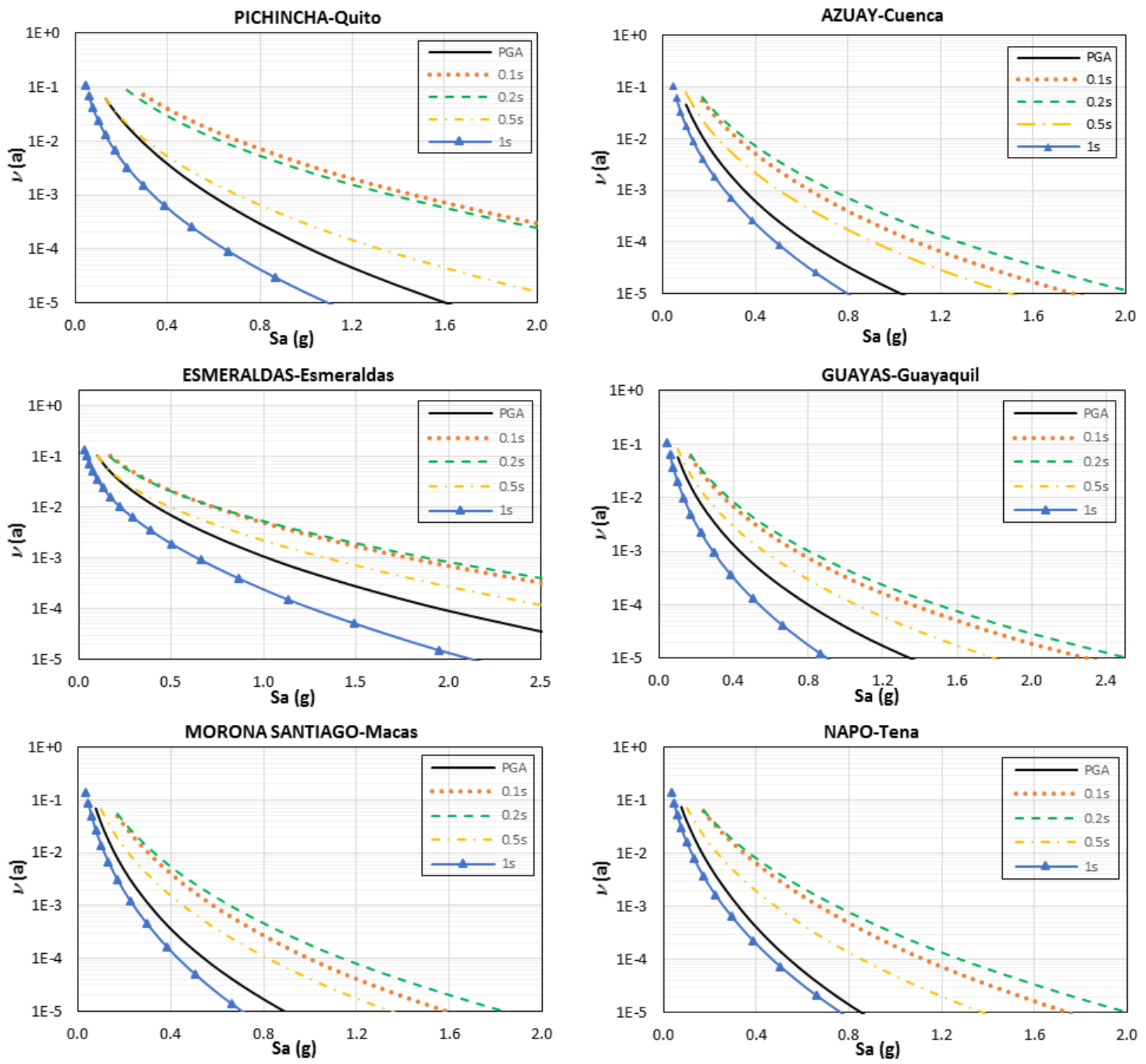

Figura 6. Curvas de peligro sísmico para ciertas provincias del Ecuador 


\section{Espectros de peligro uniforme (EPU)}

Los espectros de peligro uniforme, obtenidos a partir de las curvas de peligro sísmico, se compararon con los espectros de diseño estipulados en la norma NEC, tanto de pseudoaceleración como para desplazamiento espectral. En este estudio, los espectros de desplazamiento espectral fueron obtenidos a partir de los de pseudoaceleración aplicando las relaciones teóricas de la dinámica estructural. En la figura 7 se presentan los resultados de los EPU de pseudoaceleración y desplazamiento (Sd) para las mismas provincias expuestas en las curvas de peligro sísmico. El periodo de retorno corresponde a 475 años, y fue elegido para poder comparar los espectros obtenidos en este trabajo con los determinados en la norma NEC (en la figura 7 NEC11_Sa y NEC11_Sd corresponde a espectros de pseudoaceleración y desplazamiento, respectivamente).
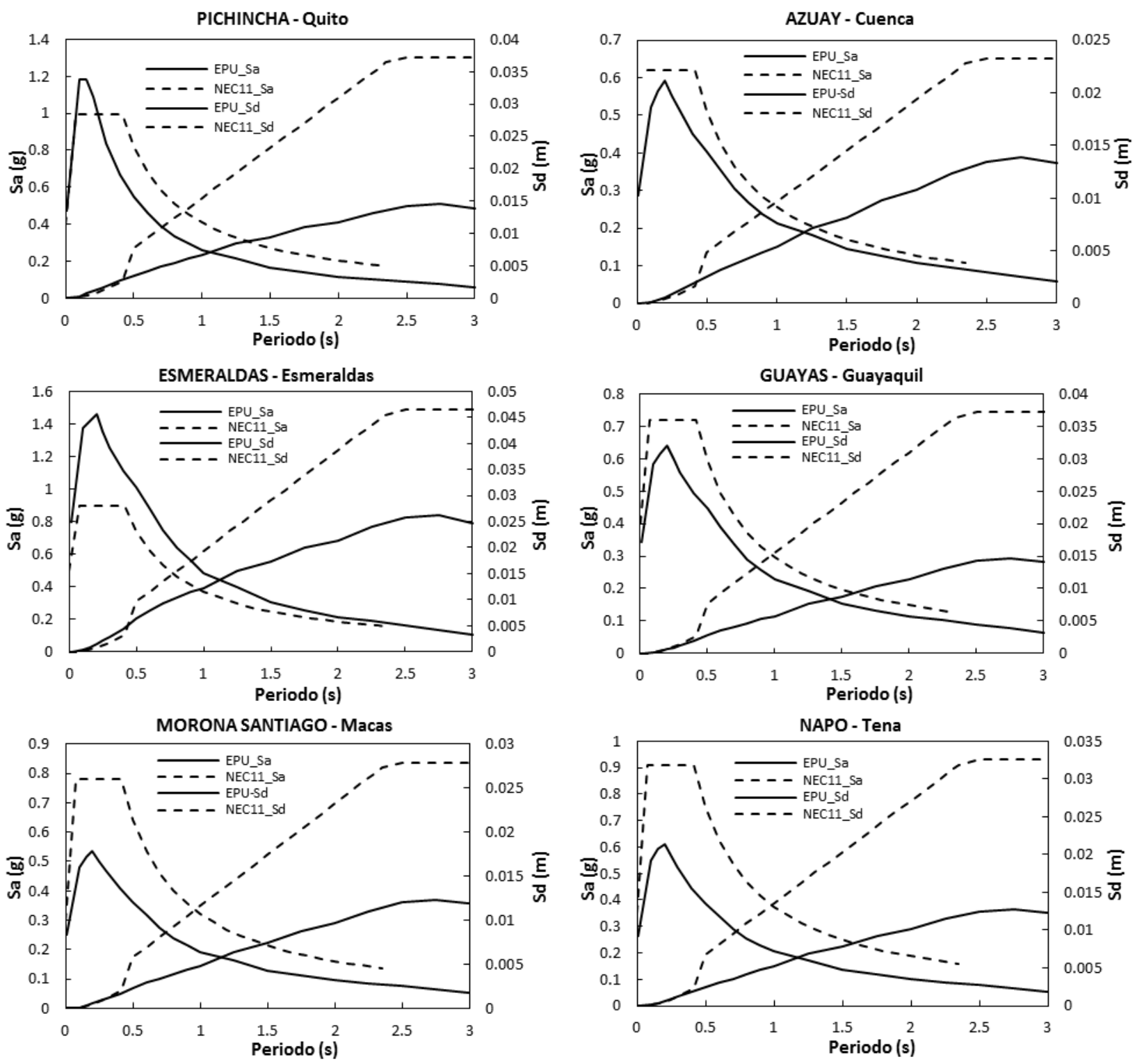

Figura 7. Espectros de peligro uniforme para ciertas provincias del Ecuador y para un periodo de retorno de 475 años 
Se pueden marcar diferencias entre los espectros de pseudoaceleración propuestos en este estudio con los de diseño estipulados en la norma NEC, dándose un mayor problema en Pichincha, Esmeraldas y Manabí (aunque esta provincia no se muestra en la figura 7), en donde el espectro de diseño es inferior a los valores de pseudoaceleración obtenidos en este trabajo, lo que podría atentar contra la seguridad estructural. Los resultados fueron comparados únicamente para roca y se considera que las diferencias se incrementarían si se empezaran a considerar efectos de sitio, ya que la norma NEC solo considera factores generales para incrementar la respuesta debido a efectos locales, los mismos que no se toman mayores a 2.25 .

Por otra parte, los espectros de desplazamiento presentan variaciones considerables, llegando a ser en ciertos casos más del doble que los EPU obtenidos en este estudio (ver figura 7). Es primordial mencionar que los espectros de la norma NEC violan los conceptos de la dinámica estructural en el aspecto que en su formulación presentan desplazamientos del terreno infinitos al obtenerlos a partir del espectro de Sa, utilizando la relación $S$ a $(T)=D(T) \Omega^{2}$, donde $\Omega$ corresponde a la frecuencia natural del sistema de un grado de libertad. Para tratar de corregir este error, en la norma NEC se incluye un espectro solo para desplazamientos (este espectro no sigue las relaciones fundamentales de la dinámica estructural), el cual presenta límites máximos y es el que se compara en la figura 7, sin embargo las diferencias siguen siendo amplias incluso al compararlas con los resultados en roca.

\section{Mapas de peligro sísmico}

Al combinar los resultados anteriores, para una región, se pueden obtener mapas de peligro sísmico, los mismos que deben estar asociados a un solo periodo de retorno. En la figura 8 se muestra los mapas de pseudoaceleración de Ecuador continental para periodos de retorno de 50, 100, 500 y 2500 años.

Los resultados del estudio de amenaza sísmica presentan dos zonas principales con alta peligrosidad. Por una parte, para $\mathrm{T}_{\mathrm{R}}$ similares a 500 años (periodo de retorno de diseño establecido en la NEC), se tienen valores elevados de intensidades espectrales en la zona de subducción (valores mayores a $0.5 \mathrm{~g}$ ), lo cual era de esperarse, en especial para la provincia de Manabí. Esto concuerda con los eventos de magnitudes mayores a 7 que se han presentado en las últimas décadas. Sin embargo, otra zona presenta intensidades cercanas a $0.45 \mathrm{~g}$, y que se debe a eventos de tipo cortical. Esta zona encierra a la ciudad de Quito, y su peligrosidad alta se puede atribuir al complejo sistema de fallas de esta zona, lo que lleva a pensar que se deben realizar estudios sísmicos a mayor detalle considerando los efectos locales ya que seguramente esto provocaría que aumenten las intensidades. 


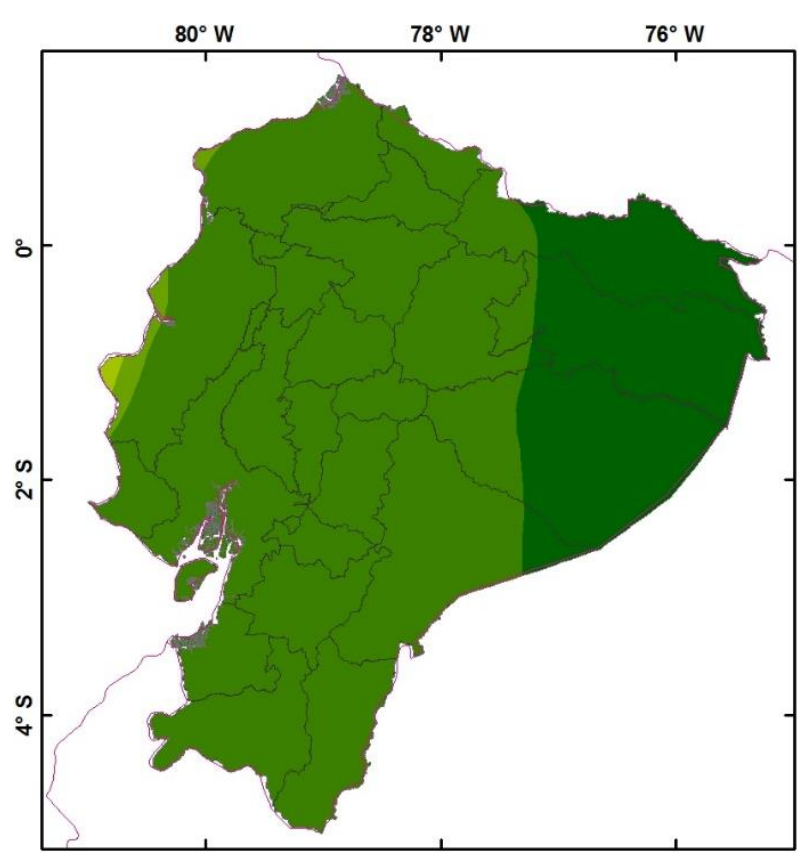

a)

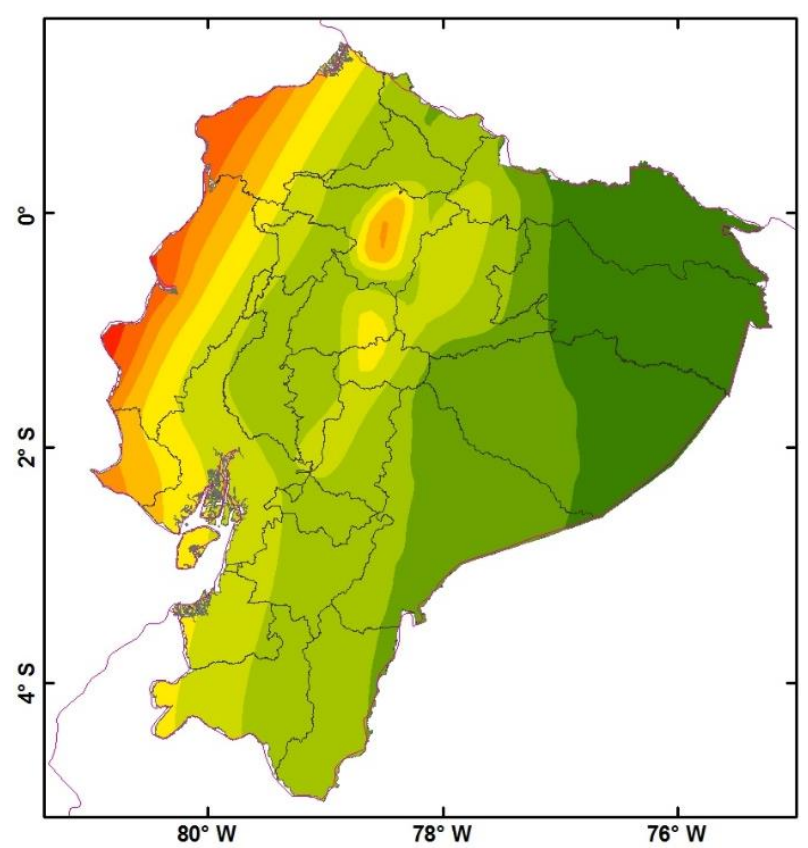

c)

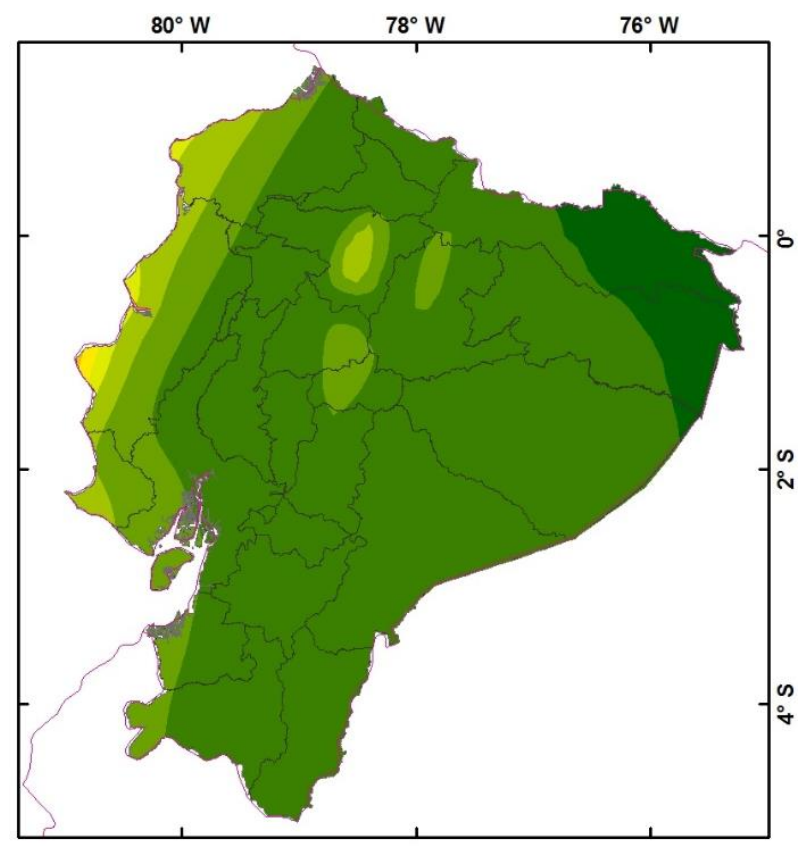

b)

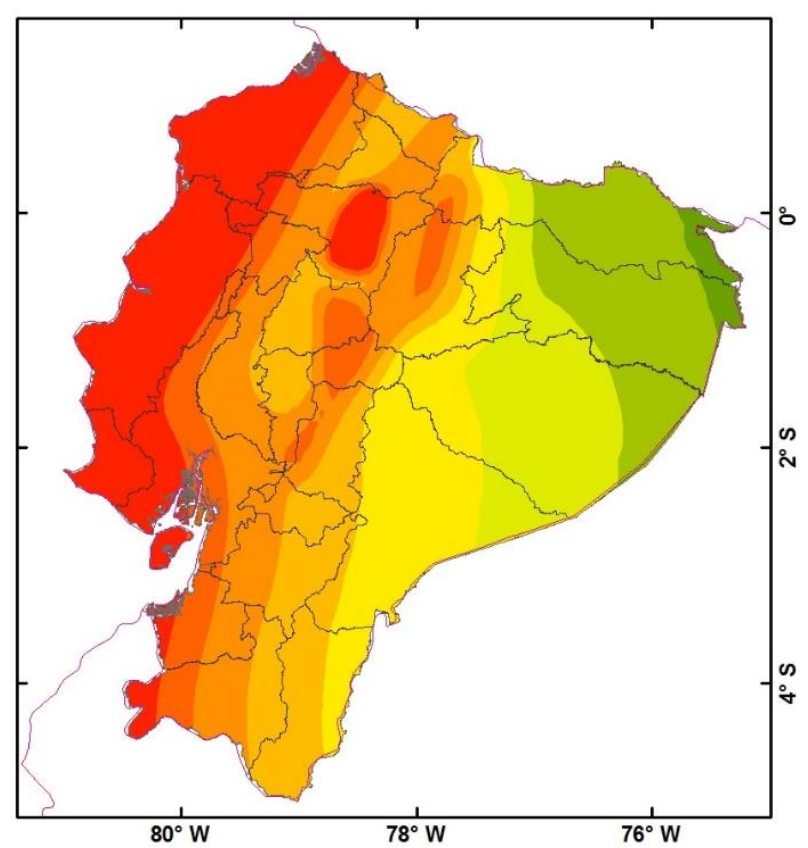

d)

\section{Simbología Temática}

\begin{tabular}{|c|c|c|c|c|c|c|c|c|c|}
\hline 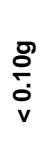 & 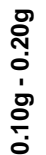 & 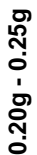 & 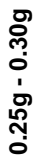 & 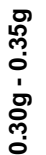 & 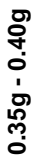 & 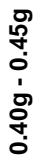 & 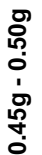 & 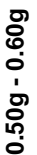 & $\begin{array}{l}\text { 웅 } \\
0 \\
0 \\
1\end{array}$ \\
\hline
\end{tabular}

Figura 8. Mapas de peligro sísmico del Ecuador para diferentes periodos de retorno: a) 50 años, b) 100 años, c) 500 años y d) 2500 años 


\section{EFECTOS DE SITIO DE LA CIUDAD DE CUENCA}

Para esta parte del estudio se utilizaron resultados de los estudios de vibración ambiental y geotecnia realizados por la Universidad de Cuenca y expuestos en la tesis de Encalada (2000), en donde se evidencian pruebas de vibración ambiental realizadas en diferentes puntos de la ciudad así como estudios de geotecnia en cuatro puntos. La zona de estudio corresponde a un área aproximada de $120 \mathrm{~km}^{2}$.

El proceso utilizado para el estudio de los efectos de sitio para la ciudad de Cuenca se puede resumir de la siguiente manera:

- Estudio geotécnico: análisis de la geología regional y de ensayos tipo SPT para estudiar las características de los estratos para las cuatro zonas en las que se dividió la ciudad.

- Monitoreo sísmico: obtención de la respuesta dinámica del suelo mediante el método de vibración ambiental.

- Efectos locales del terreno: Obtención de las funciones de transferencia, factores de amplificación y curvas de isoperiodos.

\section{Estudio geotécnico}

Se dividió a la ciudad en cuatro zonas principales, relacionadas a las características del terreno, que son Universidad de Cuenca (UC), Universidad del Azuay (UA), Parque de Miraflores (PM) y Cementerio (CM). En cada zona se realizó un ensayo tipo SPT, a través del cual se obtuvieron propiedades de los estratos del terreno, incluyendo la velocidad de onda de corte.

A partir de estos resultados, fue posible realizar un modelo unidimensional del suelo, obteniendo así las funciones de transferencia para cada zona, las cuales se muestran en la figura 9. Resultados similares se los puede encontrar en Encalada (2000). Con las funciones de transferencia se puede obtener la amplificación que sufren las ondas sísmicas debido a las características de los suelos superficiales, para cierto movimiento producido en roca.
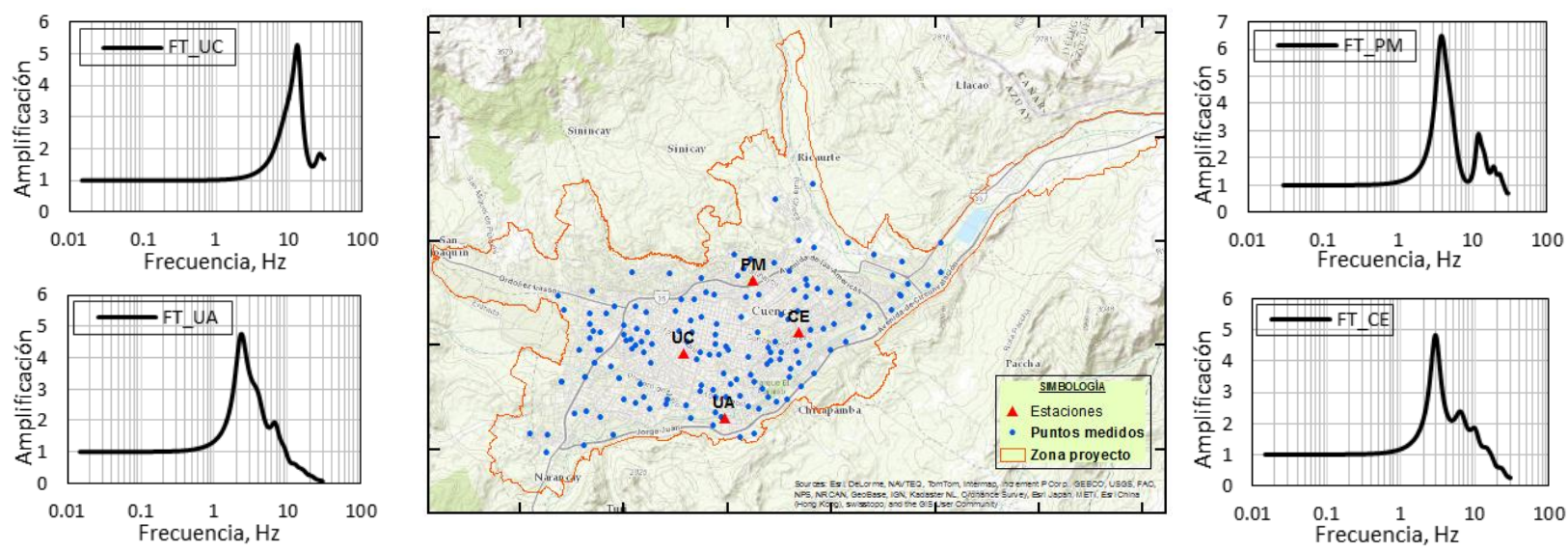

Figura 9. Funciones de transferencia para cada zona.

\section{Monitoreo sísmico}

Para la ciudad de Cuenca se cuenta con los resultados de un trabajo de vibración ambiental realizado por la Universidad de Cuenca (Encalada, 2000). Este estudio fue realizado a partir de 171 puntos de medición a lo largo de toda la ciudad. Los puntos estudiados se muestran en la figura 9. 
El tiempo de medición de las pruebas presentadas en Encalada (2000) podría no llegar a ser representativo de la respuesta del sitio, ya que en promedio se tomaron muestras de tres minutos por punto, por lo que se pudieron obviar los picos más elevados de la señal. Debido a esto existe una posibilidad que no se represente la verdadera respuesta del suelo. Sin embargo, estos puntos corresponden al único estudio de este tipo que se ha realizado en la ciudad de Cuenca. Es por esto que pese a que no se tiene una completa confiabilidad en los resultados del estudio de vibración ambiental se han tomado estos valores para el estudio de efectos de sitio presentados en este trabajo. Por lo anterior, se depuró la base de datos de los puntos del estudio tomando como base las pruebas de geotecnia presentadas (estaciones UC, UA, CE, PM, figura 9), ya que al compararlas con otros ensayos más recientes no se identifican diferencias considerables.

Para encontrar los periodos dominantes del terreno se utilizó la técnica de Nakamura que se basa en estimar la razón del espectro de Fourier entre las componentes horizontal y vertical de un mismo registro (Nakamura, 1989). La máxima amplitud del cociente H/V corresponde a la frecuencia dominante del terreno.

Para tener una malla más refinada, se utilizan los estudios de la geología local, de manera que se puedan modificar zonas en las que se consideren que se encuentran en terreno firme o en zonas de iguales condiciones a las obtenidas en las mediciones de campo. En la figura 10 se presenta el mapa de isoperiodos obtenido para la ciudad de Cuenca.

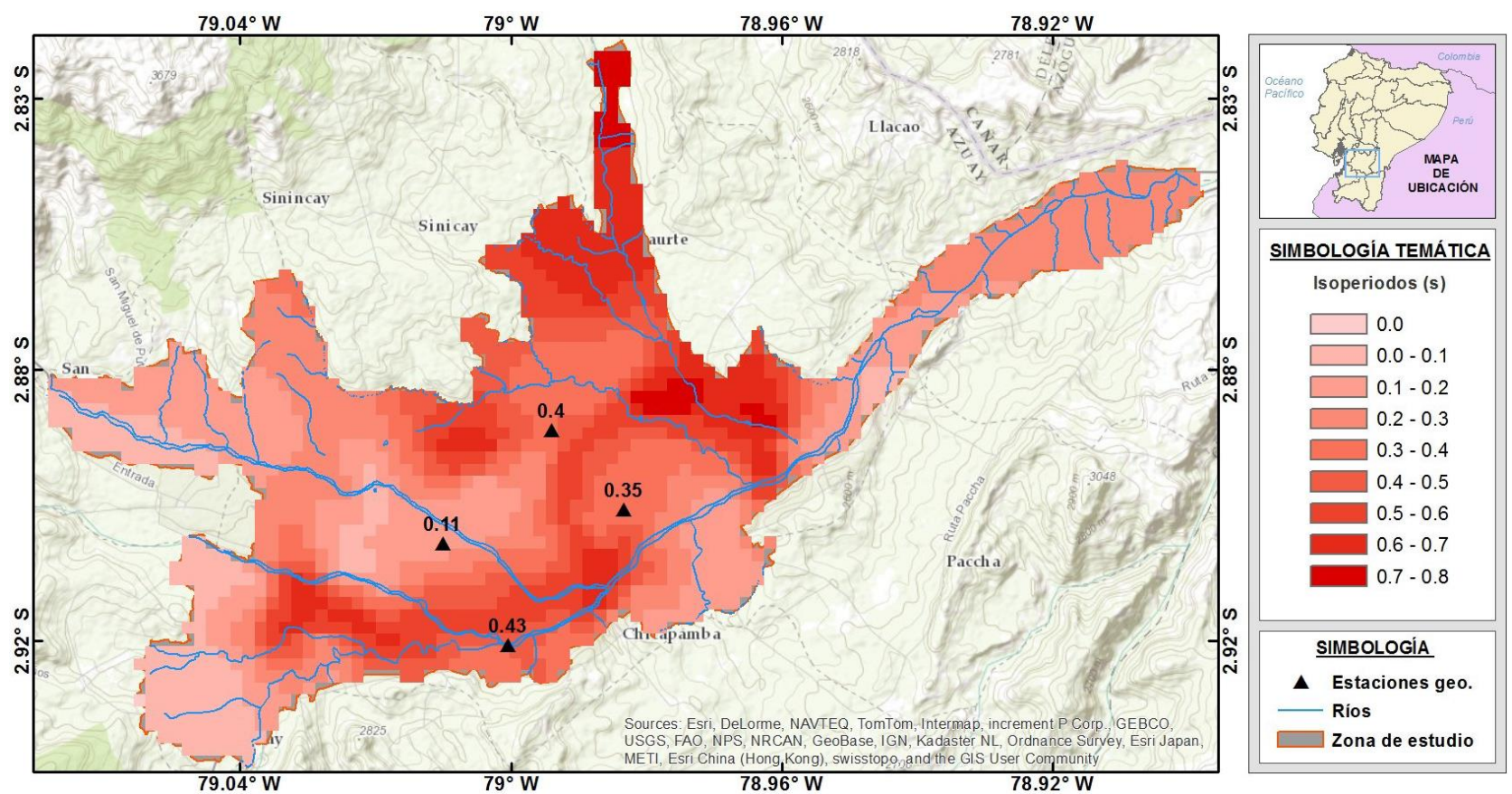

Figura 10. Mapa de isoperiodos para la ciudad de Cuenca

Para obtener los valores de las intensidades en la superficie se partió de la malla que contiene los resultados de peligro sísmico en roca para Cuenca. Posteriormente se creó una malla de las mismas dimensiones que la utilizada en el estudio de peligro sísmico en roca, en la que a cada punto le fue asignado un cociente espectral de respuesta dependiendo del periodo dominante del terreno para ese punto. La tabla 5 muestra los intervalos de periodos con su correspondiente cociente espectral de respuesta (CER). 
Tabla 5. Cocientes espectrales de respuesta, asignados a diferentes intervalos de periodos

\begin{tabular}{cc}
\hline Intervalos de periodos & CER asignado \\
\hline $0.00<\mathrm{T}<0.05$ & CER_lineal \\
$0.05<\mathrm{T}<0.15$ & CER_UC \\
& $($ ver Figura 9) \\
$0.15<\mathrm{T}<0.30$ & CER_PM \\
$0.30<\mathrm{T}<0.40$ & (ver Figura 9) \\
$0.40<\mathrm{T}<1.00$ & CER_CE \\
& (ver Figura 9) \\
CER_UA \\
\end{tabular}

Con esta nueva malla es posible obtener los valores de intensidades a nivel de superficie, aplicando la siguiente relación.

$$
S_{S}\left(w_{0}, v_{A}\right)=F T\left(w_{0}\right) S_{R}\left(w_{0}, v_{A}\right)
$$

donde, $\mathrm{S}\left(w_{0}, v_{A}\right)$ corresponde a la aceleración en suelo para cierta frecuencia $\left(w_{0}\right)$ y tasa de excedencia $\left(v_{A}\right)$. Los subíndices $S$ y $R$ corresponden a superficie y roca respectivamente. La función de transferencia para cierta frecuencia se indica como $F T\left(w_{0}\right)$. Con esta expresión se pueden obtener los resultados de la demanda afectados por los efectos de sitio, los mismos que son necesarios en la construcción del espectro de diseño para la ciudad de Cuenca.

\section{CONSTRUCCIÓN DE LOS ESPECTROS DE DISEÑO}

La forma de los espectros de diseño propuesta en esta investigación corresponde a cuatro zonas que se definen en la figura 11:

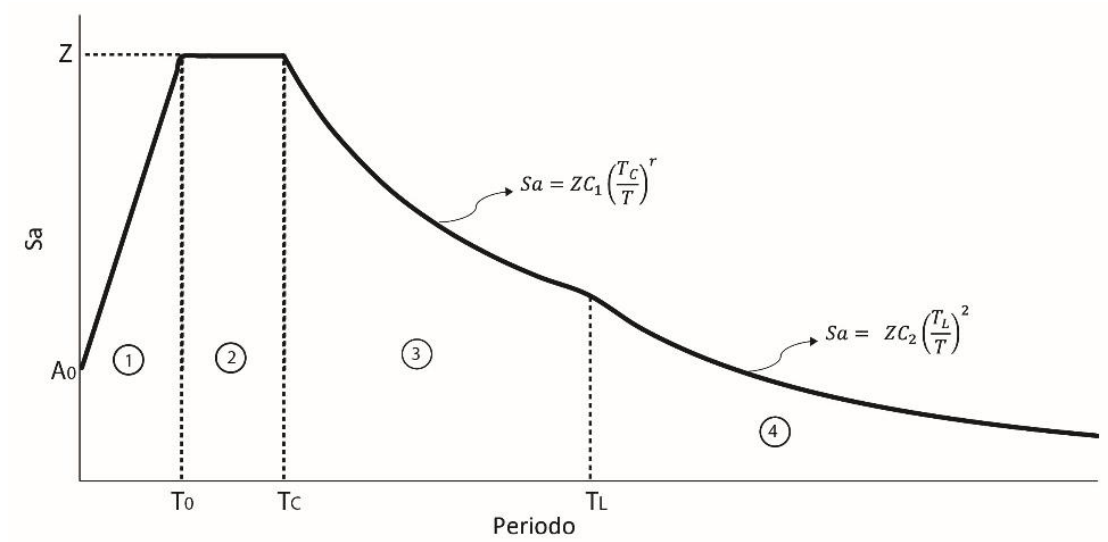

Figura 11. Forma del espectro de diseño planteada en este trabajo

- Zona 1: Zona de periodos bajos, que tiene como límite el periodo $T_{0}$. Parte del valor de $A_{0}$ que representa la aceleración máxima esperada del suelo, y crece con pendiente constante hasta $Z$ que corresponde a la aceleración espectral máxima. 
- Zona 2: Zona de periodos intermedios, parte de $T_{0}$ y termina en $T_{C}$. Valores de aceleraciones espectrales constantes e iguales a $Z$.

- Zona 3: Rama descendente controlada por la pseudovelocidad, comienza en $T_{C}$ y termina en $T_{L}$. El valor de $r$ define la forma de la caída del espectro, este valor es unitario en basamento rocoso y se incrementa en suelos blandos.

- Zona 4: Rama descendente controlada por el desplazamiento, comienza en $T_{L}$. Es inversamente proporcional al cuadrado del periodo, de manera que se pueda considerar un desplazamiento máximo del terreno.

El espectro queda definido por tres periodos $T_{0}, T_{C}$ y $T_{L}$, así como por $A_{0}, \mathrm{Z}$ y r. Las variables $C_{1}$ y $C_{2}$ están ligadas al tipo del terreno y son de ayuda para aproximar las curvas de los espectros de diseño a los resultados de los EPU. La forma de las ramas descendentes está afectada por los efectos locales del terreno, siendo esta más pronunciada en suelos blandos.

Uno de los objetivos principales del estudio de espectro de diseño es buscar un mayor detalle en la rama descendente, ya que los métodos de diseño y control de daños de las normativas actuales están ligados a los desplazamientos y derivas de piso, y el utilizar espectros sobrevalorados provocaría diseños muy conservadores. Por otra parte, ciertos reglamentos, incluyendo la NEC, violan los principios de la dinámica estructural ya que los desplazamientos máximos del terreno son considerados infinitos, y no se toma en cuenta la aceleración máxima del terreno.

La forma paramétrica del espectro es la siguiente:

- Zona 1: $0<T \leq T_{0}$

$$
S a=A_{0}+\left(Z-A_{0}\right)\left(\frac{T}{T_{0}}\right)
$$

- Zona 2: $T_{0}<T \leq T_{C}$

$$
S a=Z
$$

- Zona 3: $T_{C}<T \leq T_{L}$

$$
S a=C_{1} Z\left(\frac{T_{C}}{T}\right)^{r}
$$

- $\quad$ Zona 4: $T \geq T_{L}$

$$
S a=C_{2} Z\left(\frac{T_{L}}{T}\right)^{2}
$$

Para encontrar las variables que definen dichas formas, se obtuvieron 670 espectros de peligro uniforme dentro de los límites de la ciudad, los cuales ya se encuentran afectados por efectos de sitio; de estos se obtuvieron los valores de las siete constantes ya que se consideró, $T_{L}=2.5 \mathrm{~s}$, debido a que al analizar los EPU obtenidos se evidenció una tendencia en el cambio de curvatura del espectro en este periodo. En la figura 12 se muestran los valores característicos de cada EPU, si bien es cierto que presentan dispersión, se han asemejado a formas continuas y que corresponden a las ecuaciones 20 a 26. Los resultados se presentan solo para un periodo de retorno de 100 años. En dichas ecuaciones y figuras, el valor de Ts corresponde al periodo resonante del suelo. 


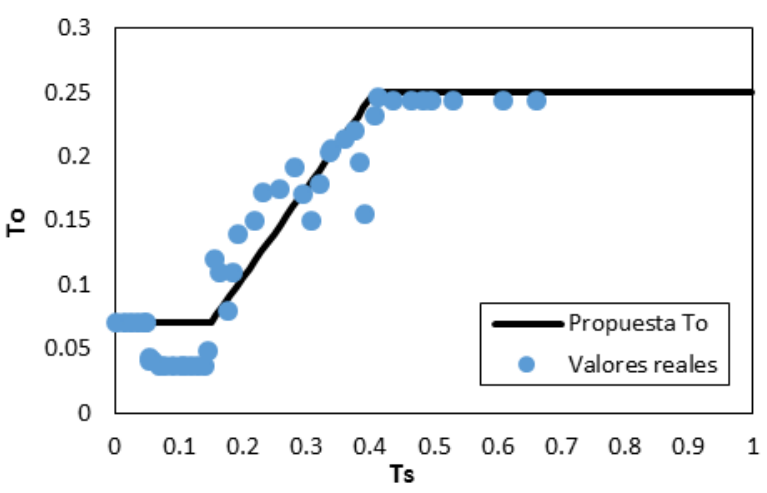

a)

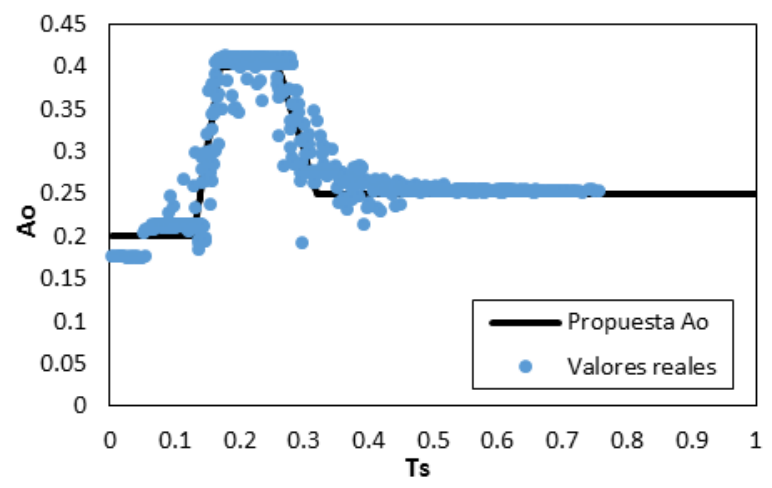

c)

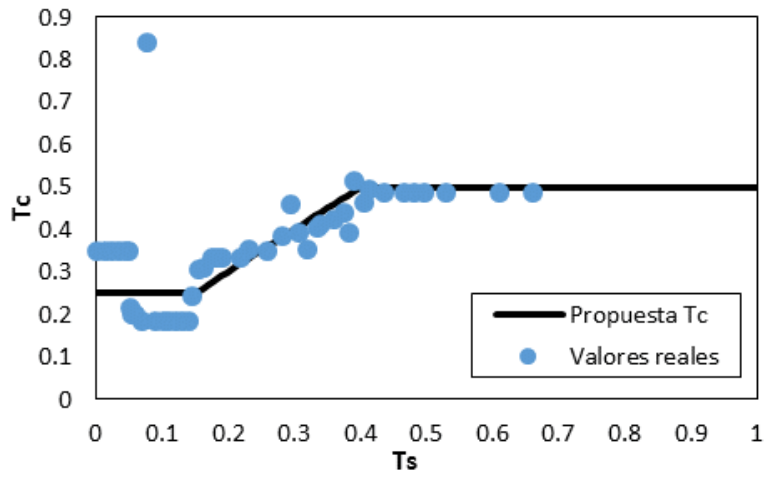

b)

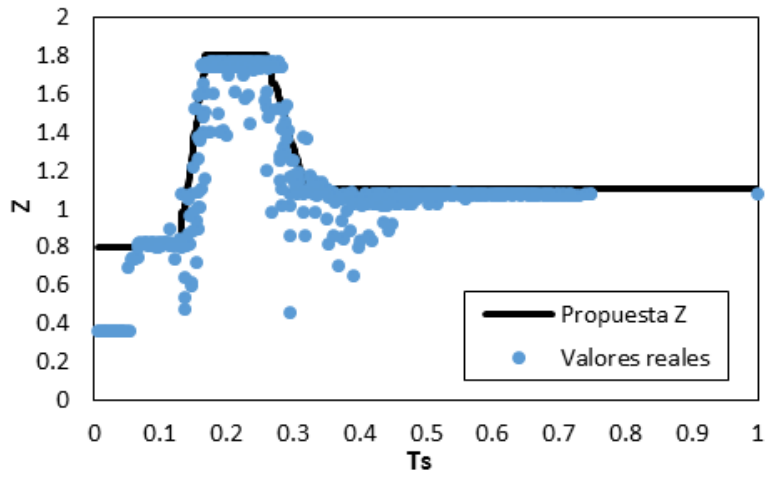

d)

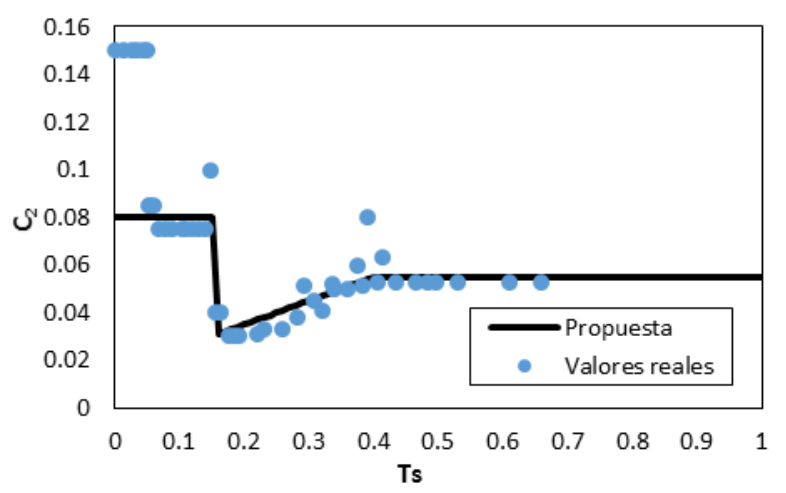

e)

Figura 12. Valores característicos de los espectros: a) $T_{0}$, b) $T_{C}$, c) $A_{0}$, d) Z y e) $C_{2}$.

$$
\begin{gathered}
T_{0}=\left\{\begin{array}{lr}
T_{0}=0.07 & \text { para } 0<T_{s} \leq 0.15 \\
T_{0}=0.7 T_{s}-0.035 & \text { para } 0.15<T_{s} \leq 0.40 \\
T_{0}=0.25 & \text { para } T_{S}>0.40
\end{array}\right\} \\
T_{C}=\left\{\begin{array}{ll}
T_{C}=0.25 & \text { para } 0.00<T_{S} \leq 0.15 \\
T_{C}=0.65 T_{s}-0.2 & \text { para } 0.15<T_{s} \leq 0.4 \\
T_{C}=0.45 & \text { para }_{S}>0.15
\end{array}\right\}
\end{gathered}
$$




$$
\begin{gathered}
A_{0}=\left\{\begin{array}{ll}
\mathrm{A}_{0}=0.2 & \text { para } 0.00<T_{s} \leq 0.13 \\
\mathrm{~A}_{0}=5.0 \mathrm{~T}_{\mathrm{s}}-0.45 & \text { para } 0.13<T_{s} \leq 0.1 \\
\mathrm{~A}_{0}=0.4 & \text { para } 0.17<T_{s} \leq 0.26 \\
\mathrm{~A}_{0}=1.05-2.5 \mathrm{~T}_{\mathrm{s}} & \text { para } 0.26<T_{s} \leq 0.32 \\
\mathrm{~A}_{0}=0.25 & \text { para }_{s}>0.32
\end{array}\right\} \\
Z=\left\{\begin{array}{ll}
Z=0.8 & \text { para } 0.00<T_{s} \leq 0.13 \\
Z=25 \mathrm{~T}_{\mathrm{s}}-2.45 & \text { para } 0.13<T_{s} \leq 0.17 \\
Z=1.8 & \text { para } 0.17<T_{s} \leq 0.26 \\
Z=4.8-11.5 \mathrm{~T}_{\mathrm{s}} & \text { para } 0.26<T_{s} \leq 0.32 \\
Z=1.1 & \text { para } T_{s}>0.32
\end{array}\right\} \\
C_{1}=\left\{\begin{array}{ll}
C_{1}=1 & \text { para } 0<T_{s} \leq 0.15 \\
C_{1}=0.85 & \text { paraT } T_{s}>0.15
\end{array}\right\} \\
C_{2}=\left\{\begin{array}{ll}
C_{2}=0.08 & \text { para } 0.00<T_{s} \leq 0.15 \\
C_{2}=0.1 T_{s}-0.015 & \text { para } 0.15<T_{s} \leq 0.4 \\
C_{2}=0.055 & \text { parat }>0.4
\end{array}\right\} \\
r=\left\{\begin{array}{ll}
r=1 & \text { para } 0<T_{s} \leq 0.15 \\
r=1.6 & \text { para } T_{s}>0.15
\end{array}\right\}
\end{gathered}
$$

Con las variables expuestas en la ecuaciones 20 a 26 quedan definidos los espectros de diseño para la ciudad de Cuenca en función del periodo del suelo $T_{S}$ y para un $T_{R}=100$ años. En la figura 13 se muestran los espectros de pseudoaceleración, pseudovelocidad ( $\mathrm{Sv}$ ) y desplazamiento espectral (Sd) para un periodo del suelo de $T_{S}=0.1 \mathrm{~s}$ y $0.4 \mathrm{~s}$. En las mismas figuras se incluyen los EPU obtenidos a partir de las curvas de peligro sísmico, así como los espectros de diseño del NEC.

Al momento de considerar efectos de sitio las diferencias con la normativa son notables, ya que la NEC utiliza factores de amplificación que se encuentran distantes a los que según este estudio se podrían llegar. La NEC propone factores máximos cercanos a 1.7, a diferencia de lo observado en Cuenca, en donde las amplificaciones pueden llegar a valores cercanos a 5. Así mismo, se ven diferencias entre los espectros propuestos en este estudio con los estipulados en las NEC. En las zonas de máxima amplificación espectral la normativa actual sobreestima los valores en aproximadamente 50\% comparándolos con los resultados de este estudio, mientras que en las zonas de periodo largo, los valores de la NEC son considerablemente mayores. En la cuarta fila de la figura 13 se compararon los espectros de desplazamientos obtenidos en este trabajo, con los calculados a partir de los espectros de pseudoaceleración de la NEC utilizando los conceptos de dinámica estructural (NEC-11_Sd_teórico); en esta comparación las diferencias son mayores y se pueden observar desplazamientos infinitos en los espectros del reglamento. 

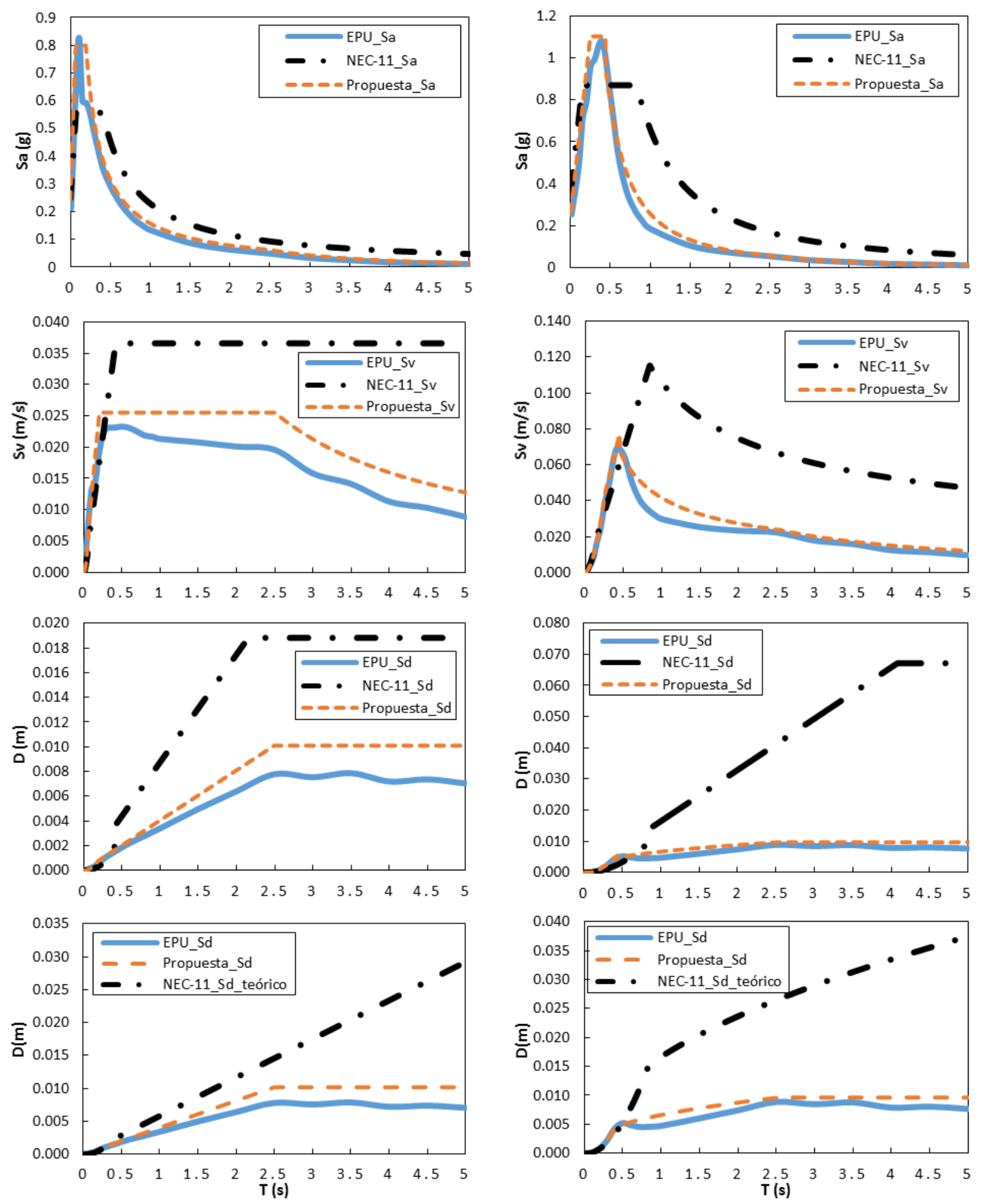

Figura 13. Comparación de los espectros de diseño propuestos y los de la NEC con los EPU obtenidos en este trabajo, para $\mathrm{Sa}, \mathrm{Sv}$ y $\mathrm{Sd}$. La columna izquierda corresponde a Ts $=0.1 \mathrm{~s}$ y la columna derecha corresponde a $\mathrm{Ts}=0.4 \mathrm{~s}$. La cuarta fila corresponde a la gráfica del EPU de desplazamiento obtenida a partir de las ecuaciones de la dinámica estructural (Sd_teórico) 


\section{SISMO EN ECUADOR DEL 16 DE ABRIL DE 2016 (M7.8)}

Mientras se trabajaba en la edición final de este trabajo ocurrió el sismo del 16 de abril de 2016 en la costa del Norte del Ecuador (Provincia de Esmeraldas). Dada la importancia de este evento, en relación a su tamaño y daños que ocasionó, se consideró adecuado incluir algunas observaciones al respecto.

La magnitud del evento fue de 7.8 (magnitud momento) cuyo epicentro se ubicó en las coordenadas $0.371^{\circ} \mathrm{N}$ y $79.940^{\circ} \mathrm{W}$ a una profundidad de $19 \mathrm{~km}$, los valores de aceleraciones máximas reportados alcanzan intensidades cercanas a los $0.6 \mathrm{~g}$ (ver figura 14). Con relación a este trabajo, el sismo se produjo dentro de la fuente de subducción 2 s, en la cual, en los últimos 110 años se han producido seis eventos con magnitud mayor a siete (31/01/1906 con $M_{w}=8.8,05 / 14 / 1942$ con $M_{w}=7.5,19 / 01 / 1958$ con $M_{w}=7.8,12 / 12 / 1979$ con $M_{w}=7.8,04 / 08 / 1998$ con $M_{w}=7.1,16 / 04 / 2016$ con $M_{w}=7.8$,). En los mapas de peligro sísmico mostrados en este trabajo (ver figura 8) se puede observar que esta zona presenta una peligrosidad sísmica, pudiendo alcanzar valores de PGA superiores a $0.6 \mathrm{~g}$, por lo que este evento se encontraría dentro de los resultados generados en este estudio.

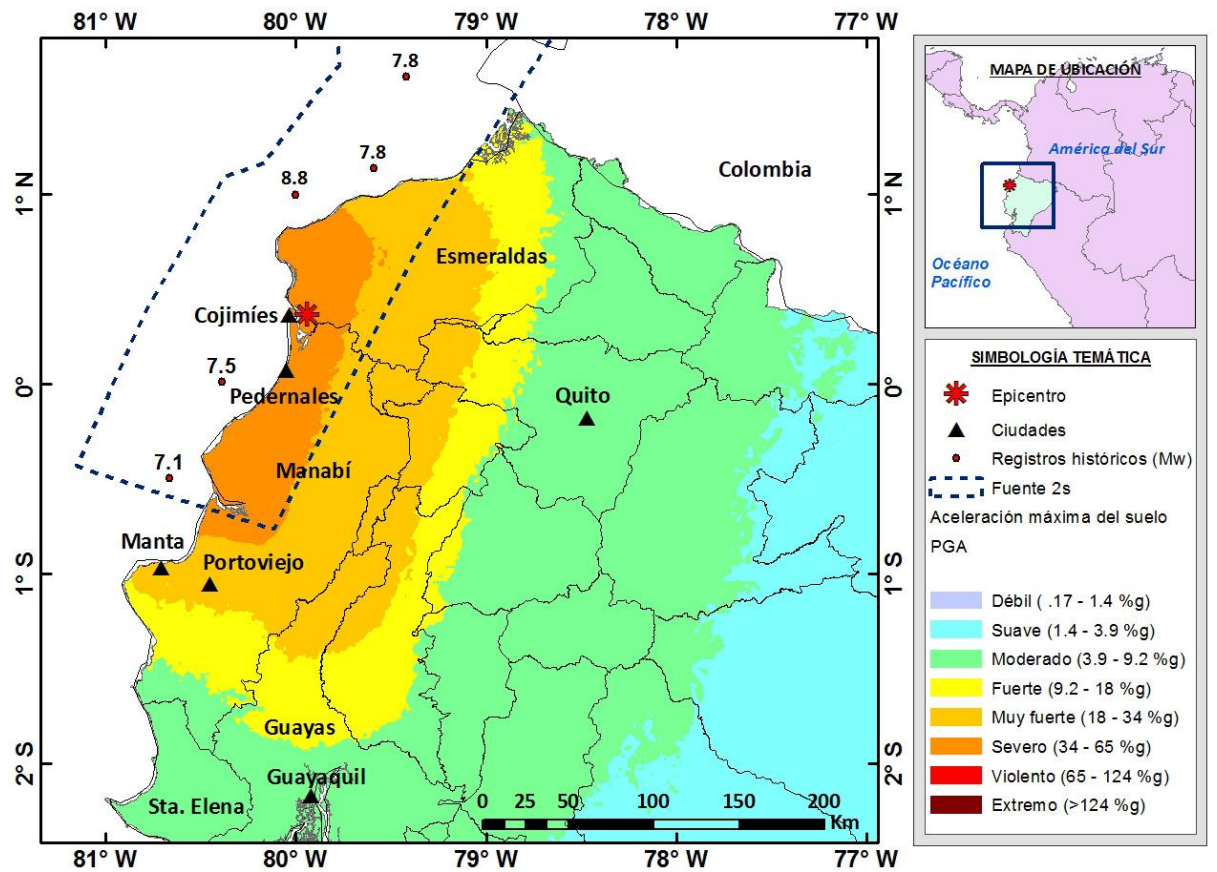

Figura 14. Mapa de aceleraciones producidas en el sismo del 16 de abril de 2016

\section{CONCLUSIONES}

Se debe continuar con el estudio de los modelos de predicción del movimiento del terreno para la región de Ecuador, ya que los que se utilizaron en este trabajo, si bien se ha probado su buen comportamiento para la región, no han sido desarrollados a partir de resultados específicos del país. Por lo que un estudio detallado de la atenuación de las ondas sísmicas contribuiría ampliamente al mejoramiento de los resultados de peligro sísmico del Ecuador.

Uno de los principales resultados de este trabajo son los parámetros de sismicidad obtenidos para cada una de las 30 fuentes sísmicas del Ecuador. A partir de éstos se podrían obtener resultados de peligro 
sísmico para diferentes tasas de excedencia y para cualquier punto de interés de la parte continental del país.

En cuanto a los resultados de la propuesta de espectros de diseño, se podría tomar como un modelo para desarrollar estudios peligro sísmico para otras ciudades del país, ya que como se mostró en los resultados obtenidos, los valores de respuestas espectrales que se podrían alcanzar superan de manera considerable a los establecidos en la normativa ecuatoriana, por lo que estudios de efectos locales serían una prioridad en el desarrollo de una normativa más detallada para el Ecuador. Como se mostró en los mapas de peligro sísmico, las zonas más sensibles serían las Costa y la zona Centro Sur del país, donde se encuentra la ciudad de Quito.

La normativa NEC, en los resultados de peligro sísmico, en ciertos casos se subestiman las aceleraciones para periodos cercanos al periodo dominante del terreno, y sobreestiman los desplazamientos asociados a la demanda sísmica en roca, en especial para las provincias de Pichincha, Manabí y Esmeraldas (figuras 7 y 13). Al considerar los efectos de sitio, esta diferencia podría incrementarse, tal como se evidenció en los resultados de amenaza sísmica de Cuenca, presentados en este trabajo.

Los graves daños que dejó el sismo del 16 de abril de 2016, dejaron en evidencia que en el Ecuador existe una deficiencia en su norma de construcción NEC, así como en su correcta aplicación, en especial en la parte correspondiente al peligro sísmico y diseño sismorresistente, por lo que estudios de este tipo deberían ser considerados en la elaboración de normativas futuras.

\section{AGRADECIMIENTOS}

Se agradece al Consejo Nacional de Ciencia y Tecnología, CONACYT, por el apoyo económico brindado para poder realizar esta investigación.

\section{REFERENCIAS}

Abrahamson, N y J Silva (1997), "Empirical Response Spectral Attenuation Relations for Shallow Crustal Earthquakes”, Seismological Research Letters, Vol. 68, pp. 94-127. DOI: 10.1785/gssrl.68.1.94.

Aguiar, R (2010), "Peligrosidad Sísmica del Ecuador y descripción de los puentes construidos sobre el estuario del río Esmeraldas, con aisladores de base FPS". Revista Internacional de Ingeniería de Estructuras, Vol. 15, No. 1, pp. 85-119.

Alvarado, A (2012), "Néotectonique et cinématique de la déformation continentable en Equateur", Institut de Sciences de la Terre de Grenoble, pp. 259.

Baker, J (2015), "Introduction to Probabilistic Seismic Hazard Analysis", White Paper Version 2.1, pp. 77.

Beauval, C, H Yepes, P Palacios, M Segovia, A Alvarado, Y Font y S Vaca (2013), "An Earthquake Catalog for Seismic Hazard Assessment in Ecuador", Bulletin of the Seismological Society of America, VOl. 103, No. 2A, pp. 773-786. DOI: 10.1785/0120120270. 
Cahuari, A y H Tavera (2007), "Cálculo de la magnitud local (ml) a partir de registros de aceleración: aplicación a sismos ocurridos en Perú”, Bol. Soc. geol. Perú, Vol. 102, pp. 117-102.

Cornell, A (1968), "Engineering Seismic Risk Analysis", Bulletin of the Seismological Society of America, Vol. 58, No. 5, pp. 1538-1606.

Cornell, A y H Vanmarcke (1969), “The major influences on seismic risk”, WCEE, Santiago de Chile, pp. 69-83.

Encalada, M (2000), “Zonificación de la ciudad de cuenca en función de los periodos dominantes", Universidad de Cuenca, pp. 328.

Esteva, L (1970), "Regionalización sísmica de México para fines de ingeniería", Serie Azul del Instituto de Ingeniería, UNAM, Publicación 246.

García, E y P Peñafiel (2000), "Estudio de Peligro Sísmico de la Región Austral”, XIII Jornadas de Ingeniería Estructural, Quito, Ecuador, Universidad Católica del Ecuador, pp. 14.

Gutenberg, B y C Richter (1944), "Frequency of Earthquakes in California", Bulletin of the Seismological Society of America, Vol. 34, No. 4, pp. 185-188.

Leonardo, M (2012), "PSM2012: Aplicación web para la difusión y consulta de datos de peligro sísmico", Tesis de maestría, Universidad Nacional Autónoma de México, pp. 98.

Maeda, K (1996), "The use of foreshocks in probabilistic prediction along the Japan and Kuril trenches", Bulletin of the Seismological Society of America, Vol. 86, No. 1A, pp. 242-254.

McGuire , R (1995), "Probabilistic Seismic Hazard Analysis and Design Earthquakes: Closing the Loop", Bulletin of the Seismological Society of America, Vol. 85, No. 5, pp. 1275-1284.

McGuire, R (2004), “Seismic Hazard and Risk Analysis”, Boulder, Colorado: EERI.

Nakamura, Y (1989), "A method for dynamic characteristic estimation of subsurface using microtremors on ground surface”, Quarterly Report of RTRI, Vol. 30, No. 1, pp. 25-33.

NEC (2014), "Norma Ecuatoriana de la Construcción. Peligro Sísmico y Diseño Sismo Resistente", Dirección de Comunicación Social, MIDUVI, Ecuador, pp. 134.

Ordaz, M y E. Pérez-Rocha (1998), "Estimation of strength-reduction factors for Elastoplastic systems: a new approach", Earthquake Eng. Struct. Dyn., Vol. 27, pp. 889-901. DOI: 10.1002/(SICI)10969845(199809)27:9<889::AID-EQE755>3.0.CO;2-W.

Ordaz, M, F Martinelli, A Aguilar, J Arboleda, C Meletti y V D'Amico (2015), "Program for computing seismic hazard: CRISIS2015 Ver 1.0”, Instituto de Ingeniería, UNAM, México.

Palacios, P y H Yepes (2011), “Analisis de la magnitud de duración”, Instituto Geofísico, Escuela Politécnica Nacional, Quito, Ecuador.

Quinde, P (2014), "Estudio de peligro sísmico del Ecuador y espectros de diseño para la ciudad de Cuenca”, Tesis de maestría, Universidad Nacional Autónoma de México, pp. 109. 
Stepp, J (1972), "Analysis of completeness of the earthquake simple in the Puget Sound area and its Effects in statical estimates of earthquake hazard". Proceedings of the microzonation conference, Washington University, pp. 897-909.

Taipe, L (2013), “Análisis de las ecuaciones de predicción de movimientos de suelo para el Ecuador, utilizando datos registrados durante el periodo 200-2011 en estaciones sísmicas de banda ancha y acelerógrafos", Tesis previa a la obtención de especialista en Geología, Escuela Politécnica Nacional, Ecuador, pp. 125.

Youngs, R, S Chiou, W Silva y J Humphrey (1997), "Strong Ground Motion Attenuation Relationship for Subduction Zone Earthquakes", Seismological Research Letters, Vol. 68, pp. 58-73. DOI: 10.1785/gssrl.68.1.58. 